\title{
Computational Mechanics of Fatigue and Life Predictions for Composite Materials and Structures
}

\author{
Jacob Fish and Qing Yu \\ Department of Civil Engineering, Mechanical and Aerospace Engineering \\ Rensselaer Polytechnic Institute, Troy, NY 12180
}

\begin{abstract}
A multiscale fatigue analysis model is developed for brittle composite materials. The mathematical homogenization theory is generalized to account for multiscale damage effects in heterogeneous media and a closed form expression relating nonlocal microphase fields to the overall strains and damage is derived. The evolution of fatigue damage is approximated by the first order initial value problem with respect to the number of load cycles. An efficient integrator is developed for the numerical integration of the continuum damage based fatigue cumulative law. The accuracy and computational efficiency of the proposed model for both low-cycle and high-cycle fatigue are investigated by numerical experimentation.
\end{abstract}

\subsection{Introduction}

In recent years several fatigue models have been developed within the framework of continuum damage mechanics (CDM) [10][12][29][35][36]. Within this framework, internal state variables are introduced to model the fatigue damage. The degradation of material response under cyclic loading is simulated using constitutive equations which couple damage cumulation and mechanical responses. The microcrack initiation and growth are lumped together in the form of the evolution of damage variables from zero to some critical value. Most of the existing CDM based fatigue damage models are based on the classical (local) continuum damage theory even though it is well known that the accumulation of damage leads to strain softening and loss of ellipticity in quasi-static problems and hyperbolicity in dynamic problems (see, for example, [1][2][5][6][19][22]). To alleviate the deficiencies inherent in the local CDM theory, a number of regularization techniques have been devised to limit the size of the strain localization zone, including the nonlocal damage theory [1][2] and gradient-dependent models [23][37]. Recent advances in CDM based theories [22][23][30][45] revealed the intrinsic links between the nonlocal CDM theory and fracture mechanics providing a possibility for building a unified framework to simulate crack initiation, propagation and overall structural failure under cyclic loading.

When applying the CDM based fatigue model to life prediction of engineering systems, the coupling between mechanical response and damage cumulation poses a major computational challenge. This is because the number of cycles to failure, especially for high-cycle fatigue, is usually as high as tens of millions or more, and therefore, it is practically not feasible to carry out a direct cycle-by-cycle simulation for the fully coupled models even with today's powerful computers. An efficient integrator, or the so-called 
"cycle jump" technique [9], for the integration of fatigue damage cumulative law must be developed for the fully coupled fatigue analysis. Several efforts have been made in this area as reported in [12] and [36].

For composite materials, the fatigue damage mechanisms are very complex primarily due to the interaction between microconstituents [14][41][42][43]. Even though numerous CDM based macro- and micromechanical models have been developed for static problems (see, for example, [1][11][20][21][25][44]), the research on the CDM based multiscale fatigue life prediction model for heterogeneous media with consistent coupling of mechanical response and damage accumulation is very limited [11].

In this paper, we develop a fatigue analysis model based on the multiscale nonlocal damage theory for composites [20] with the fatigue damage cumulative law stated on the smallest scale of interest. In Section 2 , the multiscale nonlocal damage theory based on the mathematical homogenization is summarized with emphasis on its application to fatigue of composites. Double scale asymptotic expansions of damage and displacements lead to the closed form expressions relating local (microscopic) fields to overall (macroscopic) strains and damage. In Section 3, a novel fatigue damage cumulative law is derived by extending the CDM based static damage evolution law proposed in [20]. The integration of fatigue law is approximated by the first order initial value problem with respect to the number of load cycles. Adaptive Modified Euler's method in conjunction with the step size control is used to integrate the initial value problem. Consistency adjustment procedures are introduced to ensure that the integration of the initial value problem preserves thermo-mechanical equilibrium, compatibility and constitutive equations. Computational framework, including implicit stress update procedures, consistent linearization schemes, and adaptive solution of initial value fatigue problem are presented in Section 4. In Section 5, we study the computational efficiency of the proposed multiscale fatigue model and compare its performance with respect to available test data. Discussion and future research directions conclude the manuscript.

\subsection{Multiscale Nonlocal Damage Theory for Composites}

The microstructure of a composite material is assumed to be statistically homogeneous with local periodicity. The Representative Volume Element (RVE) can be identified as shown in Figure 1, where RVE is denoted by $\Theta$. The size of RVE is assumed to be small compared to the characteristic length of macro domain so that the asymptotic homogenization applies [7][38]. Let $\boldsymbol{x}$ be the macroscopic coordinate vector in the macro domain $\Omega$ and $\boldsymbol{y} \equiv \boldsymbol{x} / \varsigma$ be the microscopic position vector in $\Theta$. $\varsigma$ denotes a very small positive number compared with the characteristic length of $\Omega$ and $\boldsymbol{y} \equiv \boldsymbol{x} / \varsigma$ is a stretched coordinate vector in the microscopic domain. Due to the high heterogeneity in the microstructure, the local oscillations exist for all the mechanical quantities. In this respect, all quantities have two explicit dependences: one on the macroscopic level $\boldsymbol{x}$ and the second one, on the level of microconstituents $\boldsymbol{y} \equiv \boldsymbol{x} / \boldsymbol{\varsigma}$. Using the classical nomenclature, any Y-periodic function $f$ can be represented as

$$
f^{\varsigma}(x) \equiv f(x, y(x))
$$

where superscript $\varsigma$ denotes the Y-periodic function $f$. 


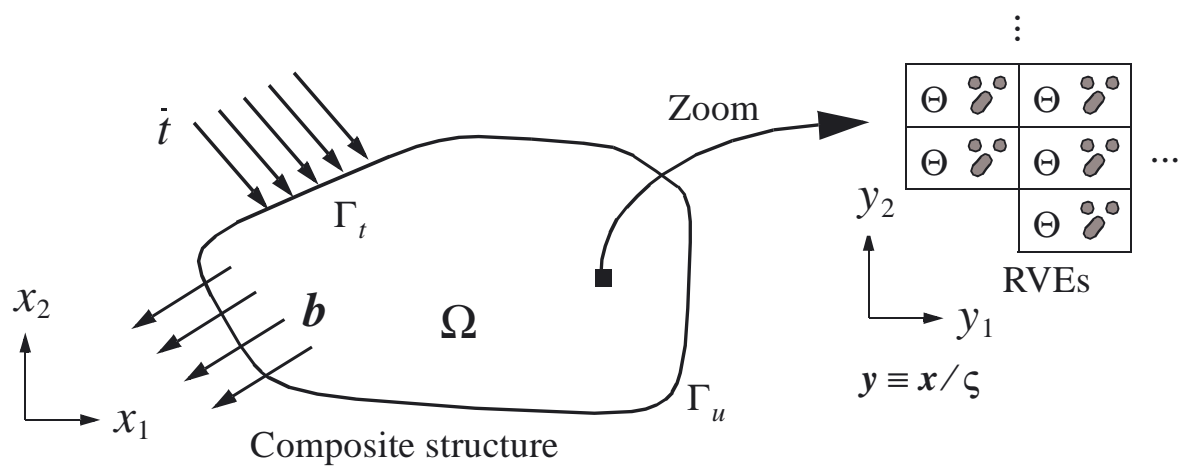

FIGURE 1. Macroscopic and Microscopic Structures

To model fatigue damage, we define a scalar damage variable $\omega^{\varsigma}$ as a function of microscopic and macroscopic position vectors, i.e., $\omega^{\varsigma}=\omega(x, y)$. The constitutive equation on the microscale is derived from the strain-based continuum damage theory. Following [39], the free energy density for has the form of

$$
\Psi\left(\omega^{\varsigma}, \varepsilon_{i j}^{\varsigma}\right)=\left(1-\omega^{\varsigma}\right) \Psi_{e}\left(\varepsilon_{i j}^{\varsigma}\right)
$$

where $\omega^{\varsigma} \in[0,1)$ is a scalar damage variable on the microscale; $\Psi_{e}\left(\varepsilon_{i j}^{\varsigma}\right)$ is elastic free energy density function given as $\Psi_{e}\left(\varepsilon_{i j}^{\varsigma}\right)=\frac{1}{2} L_{i j k l} \varepsilon_{i j}^{\zeta} \varepsilon_{k l}^{\varsigma}$. Throughout this paper, the summation convention for repeated subscripts is employed, except for the subscripts $x$ and $y$. We assume that the micro-constituents possess homogeneous properties and satisfy the following boundary value problem:

$$
\begin{array}{ll}
\sigma_{i j, x_{j}}^{\varsigma}+b_{i}=0 & \text { in } \Omega \\
\sigma_{i j}^{\varsigma}=\left(1-\omega^{\varsigma}\right) L_{i j k l} \varepsilon_{k l}^{\varsigma} & \text { in } \Omega \\
\varepsilon_{i j}^{\varsigma}=u_{\left(i, x_{j}\right)}^{\varsigma} & \text { in } \Omega \\
u_{i}^{\varsigma}=\bar{u}_{i} & \text { on } \Gamma_{u} \\
\sigma_{i j}^{\varsigma} n_{j}=\dot{t}_{i} & \text { on } \Gamma_{t}
\end{array}
$$

where $\sigma_{i j}^{\varsigma}$ and $\varepsilon_{i j}^{\varsigma}$ are components of stress and strain tensors; $L_{i j k l}$ denotes the elastic constitutive tensor components; $b_{i}$ is a body force assumed to be independent of $\boldsymbol{y} ; u_{i}^{\varsigma}$ denotes components of the displacement vector; $\Omega$ is the macroscopic domain of interest with boundary $\Gamma ; \Gamma_{u}$ and $\Gamma_{t}$ are the boundary portions where displacements $\bar{u}_{i}$ and tractions $\dot{t}_{i}$ are prescribed, respectively, such that $\Gamma_{u} \cap \Gamma_{t}=\varnothing$ and $\Gamma=\Gamma_{u} \cup \Gamma_{t} ; n_{i}$ denotes the normal vector component on $\Gamma$. We assume that the interface between the phases is perfectly bonded, i.e. $\left[\sigma_{i j}^{\varsigma_{j}} \hat{n}_{j}\right]=0$ and $\left[u_{i}\right]=0$ at the interface, $\Gamma_{i n t}$, where $\hat{n}_{i}$ denotes the normal vector to $\Gamma_{\text {int }}$ and [•] is a jump operator; the subscript pairs with parenthesizes denote the symmetric gradients defined as $u \varsigma_{\left.i, x_{j}\right)} \equiv\left(u_{i, x_{j}}^{\varsigma}+u_{j, x_{i}}^{\varsigma}\right) / 2$. 
Since the discretization with the mesh size comparable to the scale of microscopic constituents is computationally prohibitive, mathematical homogenization theory is employed to account for microstructural effects without explicitly representing the details of microstructure in the global analysis. This is accomplished by approximating the displacement field, $u_{i}^{\mathcal{S}}(\boldsymbol{x})=u_{i}(\boldsymbol{x}, \boldsymbol{y})$ and the damage variable, $\omega^{\varsigma}(\boldsymbol{x})=\omega(\boldsymbol{x}, \boldsymbol{y})$, in terms of the double-scale asymptotic expansions on $\Omega \times \Theta$ :

$$
\begin{aligned}
& u_{i}(\boldsymbol{x}, \boldsymbol{y}) \approx u_{i}^{0}(\boldsymbol{x}, \boldsymbol{y})+\varsigma u_{i}^{1}(\boldsymbol{x}, \boldsymbol{y})+\ldots \\
& \omega(\boldsymbol{x}, \boldsymbol{y}) \approx \omega^{0}(\boldsymbol{x}, \boldsymbol{y})+\varsigma \omega^{1}(\boldsymbol{x}, \boldsymbol{y})+\ldots
\end{aligned}
$$

where the superscripts denote the order of terms in the asymptotic expansion. With these expansions, we have developed a non-local damage theory for brittle composites in [20]. In the rest of this section, we merely present the major results which are closely related to the fatigue problem of interest in this paper. The complete derivation is referred to [20].

By inserting the expansion (8) and (9) into the boundary value problem (3)-(7), we can obtain a set of equilibrium equations for the various orders of $\varsigma$ starting from $\varsigma^{-2}$. The solution to these equations gives the asymptotic expansion of the strain field in RVE as

$$
\varepsilon_{i j}(\boldsymbol{x}, \boldsymbol{y})=A_{i j m n}(\boldsymbol{y}) \bar{\varepsilon}_{m n}(\boldsymbol{x})+G_{i j m n}(\boldsymbol{y}) d_{m n}^{\omega}(\boldsymbol{x})+O(\varsigma)
$$

where $\bar{\varepsilon}_{m n}(\boldsymbol{x})$ is the elastic strain in macro domain and $d_{m n}^{\omega}(\boldsymbol{x})$ is a damage-induced macroscopic strain; $A_{i j m n}(y)$ is the so-called elastic strain concentration function [25] given by

$$
A_{i j m n}=I_{i j m n}+G_{i j m n} ; \quad I_{i j m n}=\frac{1}{2}\left(\delta_{i m} \delta_{j n}+\delta_{i n} \delta_{j m}\right)
$$

where $\delta_{m k}$ is Kronecker delta; $G_{i j m n}(\boldsymbol{y})$ is termed as the local distribution function of damage-induced strain, which can be obtained by solving a linear boundary value problem in $\Theta$ with Y-periodic boundary conditions, i.e.

$$
\left\{L_{k l i j}\left(I_{i j m n}+H_{\left(i, y_{j}\right) m n}(\boldsymbol{y})\right)\right\}_{, y_{l}}=0
$$

where $H_{i m n}(\boldsymbol{y})$ is a Y-periodic third rank tensor with symmetry $H_{\text {imn }}=H_{\text {inm }}$, and

$G_{i j m n}(\boldsymbol{y})=H_{\left(i, y_{j}\right) m n}(\boldsymbol{y})$. The damage-induced strain $d_{m n}^{\omega}(\boldsymbol{x})$ can be related to the elastic strain in macro domain through a fourth rank tensor

$$
d_{m n}^{\omega}(\boldsymbol{x})=D_{k l m n}(\boldsymbol{x}) \bar{\varepsilon}_{m n}(\boldsymbol{x})
$$

where $D_{k l m n}(\boldsymbol{x})$, which is determined by the damage state in each microconstituents, represents the influence of the fatigue damage cumulation on the macroscopic response. Following [20], we have 


$$
D_{k l m n}(x)=\left(I_{k l s t}-\sum_{\eta=1}^{n} B_{k l s t}^{(\eta)} \omega^{(\eta)}(x)\right)^{-1}\left(\sum_{\eta=1}^{n} C_{s t m n}^{(\eta)} \omega^{(\eta)}(x)\right)
$$
where $\eta$ denotes different phases in RVE such that $\underset{\eta=1}{\cup} \Theta^{(\eta)}=\Theta ; \omega^{(\eta)}(\boldsymbol{x})$ represents the phase average
damage; $B_{i j k l}^{(\eta)}$ and $C_{i j k l}^{(\eta)}$ are given by

$$
\begin{gathered}
B_{i j k l}^{(\eta)}=\frac{1}{|\Theta|}\left(\tilde{L}_{i j m n}-\bar{L}_{i j m n}\right)^{-1} \int_{\Theta^{(\eta)}} G_{s t m n} L_{s t p q} G_{p q k l} d \Theta \\
C_{i j k l}^{(\eta)}=\frac{1}{|\Theta|}\left(\tilde{L}_{i j m n}-\bar{L}_{i j m n}\right)^{-1} \int_{\Theta^{(\eta)}} G_{s t m n} L_{s t p q} A_{p q k l} d \Theta \\
\tilde{L}_{i j m n}=\frac{1}{|\Theta|} \int_{\Theta} L_{i j m n} d \Theta \quad \text { and } \quad \bar{L}_{i j k l}=\frac{1}{|\Theta|} \int_{\Theta} L_{i j m n} A_{m n k l} d \Theta
\end{gathered}
$$

where $|\Theta|$ is the volume of a RVE and we notice that $\bar{L}_{i j k l}$ is the homogenized elastic stiffness tensor[33]. According to (13) and (14), it is clear that the damage-induced strain $d_{m n}^{\omega}(\boldsymbol{x})$ vanishes when the microstructure is free of damage.

After solving for the local strain field in the RVE, the homogenized field can be obtained by the phase average process. By integrating (10) over $\Theta^{(\eta)}$ and making use of (13), we have

$$
\varepsilon_{i j}^{(\eta)}=\frac{1}{\mid \Theta^{(\eta) \mid}} \int_{\Theta^{(\eta)}} \varepsilon_{i j} d \Theta=A_{i j k l}^{(\eta)} \bar{\varepsilon}_{k l}+G_{i j k l}^{(\eta)} D_{k l m n} \bar{\varepsilon}_{m n}+O(\varsigma)
$$

where

$$
A_{i j k l}^{(\eta)}=\frac{1}{\mid \Theta^{(\eta) \mid}} \int_{\Theta^{(\eta)}} A_{i j k l} d \Theta \quad \text { and } \quad G_{i j k l}^{(\eta)}=\frac{1}{\left|\Theta^{(\eta) \mid}\right|} \int_{\Theta^{(\eta)}} G_{i j k l} d \Theta
$$

The constitutive equating for the phase average field can be expressed as

$$
\sigma_{i j}^{(\eta)}=\left(1-\omega^{(\eta)}\right) L_{i j m n}^{(\eta)} \varepsilon_{m n}^{(\eta)}
$$

where $\sigma_{i j}^{(\eta)}$ is the phase average stress, and the overall homogenized stress field turns into

$$
\bar{\sigma}_{i j}=\sum_{\eta=1}^{n} v^{(\eta)} \sigma_{i j}^{(\eta)}
$$

where $v^{(\eta)}$ is the volume fractions for phase $\Theta^{(\eta)}$ in RVE satisfying $\sum_{\eta=1}^{n} v^{(\eta)}=1$. The phase free energy density corresponding to the nonlocal constitutive equation (20) is given as 


$$
\Psi^{(\eta)}\left(\omega^{(\eta)}, \varepsilon_{i j}^{(\eta)}\right)=\frac{1}{2}\left(1-\omega^{(\eta)}\right) L_{i j m n}^{(\eta)} \varepsilon_{m n}^{(\eta)} \varepsilon_{i j}^{(\eta)}
$$

and the corresponding phase damage energy release rate and the energy dissipation inequality [9][39] applied to the phase average field can be expressed as

$$
\begin{gathered}
Y^{(\eta)}=-\frac{\partial \Psi^{(\eta)}}{\partial \omega^{(\eta)}}=\frac{1}{2} L_{i j m n}^{(\eta)} \varepsilon_{m n}^{(\eta)} \varepsilon_{i j}^{(\eta)} \\
Y^{(\eta)} \dot{\omega}^{(\eta)} \geq 0
\end{gathered}
$$

It should be noted that the nonlocal character of the phase average damage $\omega^{(\eta)}$ and the constitutive equation (20) has been proved in [20]. This important feature validates our homogenization theory for simulating the damage evolution in composite materials [1][2][5][23][30][37]. In the next two sections, a fatigue damage cumulative law for the two-phase composites as well as computational framework, including implicit stress update procedures, consistent linearization schemes, and adaptive integration of the initial value fatigue problem are described.

\subsection{Fatigue Damage Cumulative Law}

In cyclic fatigue process, the damage accumulation is usually dependent of previous damage history, loading sequence and frequency, material properties, and environmental effects. A large number of purely phenomenological fatigue damage cumulative laws have been proposed since the middle of this century [15][16]. Within the framework of continuum damage mechanics, several fatigue damage laws for homogeneous materials were developed recently (see, for example, [3][4][9][12][13][16][29][36]). In [35][42], the application of CDM to the fatigue of heterogeneous media is also explored. An obvious advantage of these CDM based damage cumulative laws is their consistency with continuum mechanics, with which a unified fatigue analysis model could be developed for numerical simulation. In this section, a new CDM based fatigue damage cumulative law for composites is developed following the scheme proposed in [29].

In our previous work [20], we defined the nonlocal "static" damage variable $\omega^{(\eta)}$ as an increasing function of nonlocal phase deformation history parameter, $\kappa^{(\eta)}$, which characterizes the maximum deformation state experienced in the local neighborhood throughout the loading history. $\kappa^{(\eta)}$ represents the evolving boundary of a reversible domain, i.e., all strain states are either within this domain or on the boundary of this domain. The evolution of the nonlocal phase static damage at a given time can be expressed as

$$
\left.\omega^{(\eta)}(x, t)=\Phi\left(<\kappa^{(\eta)}(x, t)-\bar{\vartheta}_{i n i}^{(\eta)}\right\rangle_{+}\right) \quad \text { and } \quad \frac{\left.\partial \Phi\left(<\bar{\vartheta}^{(\eta)}(x, t)-\bar{\vartheta}_{i n i}^{(\eta)}\right\rangle_{+}\right)}{\partial \kappa^{(\eta)}} \geq 0
$$

where $\omega^{(\eta)} \in[0,1)$; the operator $\left\langle>_{+}\right.$denotes the positive part, i.e. $<\bullet>_{+}=\sup \{0, \bullet\}$; the phase deformation history parameter $\kappa^{(\eta)}$ is determined from the evolution of the phase damage equivalent strain, denoted by $\vartheta$ 


$$
\kappa^{(\eta)}(\boldsymbol{x}, t)=\max \left\{\bar{\vartheta}^{(\eta)}(\boldsymbol{x}, \tau) \mid(\tau \leq t), \bar{\vartheta}_{\text {ini }}^{(\eta)}\right\}
$$

where $\bar{\vartheta}_{\text {ini }}$ represents the threshold value of damage equivalent strain prior to the initiation of phase damage; $\vartheta^{-(\eta)}$ is defined as the square root of the phase damage energy release rate [39]

$$
\bar{\vartheta}^{(\eta)}=\sqrt{Y^{(\eta)}}=\sqrt{\frac{1}{2} L_{i j k l}^{(\eta)} \varepsilon_{i j}^{(\eta)} \varepsilon_{k l}^{(\eta)}}
$$

Since $L_{i j k l}^{(\eta)}$ is assumed to be a positive definite fourth order tensor it follows that $Y^{(\eta)} \geq 0$ and consequently, $\quad \dot{\omega}^{(\eta)} \geq 0$ must hold due to the energy dissipation inequality in (24).

To generalize the above static damage evolution law to cyclic fatigue, we first reformulate the static law. The key is to preserve the irreversible character of the internal state variable $\omega^{(\eta)}$ and to relate it to the nonlocal phase deformation history. Without loss of generality this can be accomplished by reformulating (25) and (26) as

$$
\begin{gathered}
\hat{\omega}^{(\eta)}(x, t)=\Phi\left(\left\langle\bar{\vartheta}^{(\eta)}(x, t)-\bar{\vartheta}_{i n i}^{(\eta)}\right\rangle_{+}\right) \quad \text { and } \quad \frac{\partial \Phi\left(\left\langle\bar{\vartheta}^{(\eta)}(x, t)-\bar{\vartheta}_{i n i}^{(\eta)}\right\rangle_{+}\right)}{\partial \bar{\vartheta}^{(\eta)}} \geq 0 \\
\omega^{(\eta)}(x, t)=\max \left\{\hat{\omega}^{(\eta)}(x, \tau) \mid(\tau \leq t)\right\}
\end{gathered}
$$

where $\hat{\omega}^{(\eta)} \in[0,1)$ is termed here as a nonlocal "pseudo-damage" parameter. By setting the so-called "gauge function" [29] as

$$
f=\hat{\omega}^{(\eta)} / \omega^{(\eta)}=\Phi^{(\eta)} / \omega^{(\eta)}
$$

the rate form of the static phase damage evolution law (29) turns into

$$
\dot{\omega}^{(\eta)}(x, t)= \begin{cases}0 & f<1 \\ \left.\frac{\partial \Phi^{(\eta)}}{\partial \bar{\vartheta}^{(\eta)}}<\dot{\vartheta}^{(\eta)}\right\rangle_{+} & f=1\end{cases}
$$

where $\left.\Phi^{(\eta)} \equiv \Phi\left(<\bar{\vartheta}^{(\eta)}(x, t)-\bar{\vartheta}_{\text {ini }}^{(\eta)}\right\rangle_{+}\right) \quad$ can be expressed as an arctangent form damage evolution proposed in [20]: 


$$
\Phi^{(\eta)}=\frac{\operatorname{atan}\left[\alpha^{(\eta)}\left(\frac{\left\langle\bar{\vartheta}^{(\eta)}-\bar{\vartheta}_{i n i}^{(\eta)}\right\rangle_{+}}{-(\eta)}\right)-\beta^{(\eta)}\right]+\operatorname{atan}\left(\beta^{(\eta)}\right)}{\frac{\pi}{2}+\operatorname{atan}\left(\beta^{(\eta)}\right)}
$$

where $\alpha^{(\eta)}, \beta^{(\eta)}, \vartheta_{0}^{-(\eta)}$ are material constants; $\vartheta_{0}^{(\eta)}$ denotes the threshold of the strain history parameter beyond which the damage will develop very quickly. The calibration of these parameters can be performed by using the quasi-static uniaxial loading test with the specimen made of phase materials [27]. The phase endurance limit $\bar{\vartheta}_{\text {ini }}^{(\eta)}$ can also be calibrated by uniaxial tests while the triaxial stress test may give better results [4].

According to [29][39], it can be proved that gauge function $f$ has the classic yield surface properties. Indeed, with the definition (31), there exists a closed reversible domain in the strain space bounded by the gauge function such that the damage does not increase from any interior point but may develop from the state on the boundary. The damage loading/unloading (inelastic/elastic) condition for any state on the boundary is determined by the sign of $\dot{\vartheta}^{(\eta)}$. From (28) and (31) it can be seen that $\operatorname{sgn}\left(\dot{\vartheta}^{(\eta)}\right)=\operatorname{sgn}(\dot{f})$. To extend this static damage evolution law to account for the fatigue damage cumulation, Marigo [29] proposed to drop the yield surface concept and to replace it by an irreversible loading/unloading concept. In this respect, the boundary of the strain space domain is assumed to be fixed and the strain state is permitted to penetrate it in the loading history, and accordingly, an extension of (31) along the lines of the power law in viscoplasticity [34] is in the form of

$$
\dot{\omega}^{(\eta)}(x, t)=\left\{\begin{array}{cc}
0 & -(\eta)<\bar{\vartheta}_{\text {ini }}^{(\eta)} \\
{\left[\frac{\Phi^{(\eta)}}{\omega^{(\eta)}}\right]^{\gamma^{(\eta)}} \frac{\partial \Phi^{(\eta)}}{\partial \bar{\vartheta}^{(\eta)}}\left\langle\dot{\bar{\vartheta}}^{(\eta)}\right\rangle_{+}} & \bar{\vartheta}^{(\eta)} \geq \bar{\vartheta}_{\text {ini }}^{(\eta)}
\end{array}\right.
$$

where $\bar{\vartheta}_{\text {ini }}(\eta)$ can be interpreted as the endurance domain within which the change of strain state along any loading path does not lead to the growth of damage; $\gamma^{(\eta)}$ is a stress dependent parameter. When $\gamma^{(\eta)} \rightarrow \infty$, the fatigue damage power law reduces to the static damage law (31) in the sense that the damage evolution is controlled by the value of $f=\Phi^{(\eta)} / \omega^{(\eta)}$. The difference between the two models is schematically illustrated in Figure 2. 


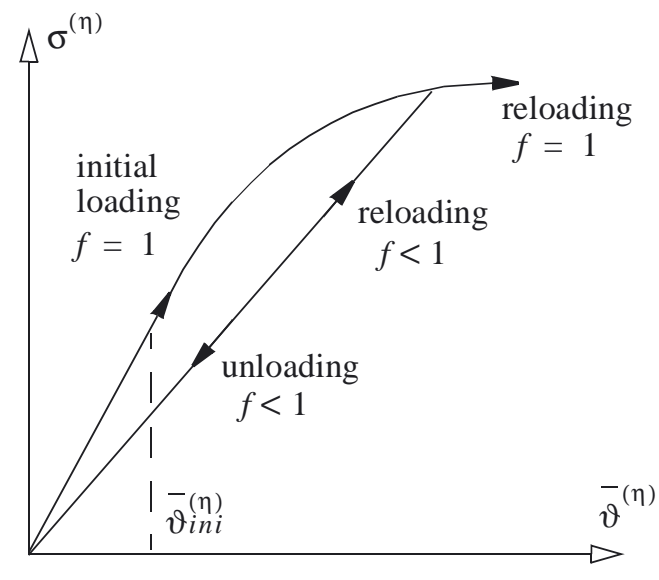

Static Damage Cumulation

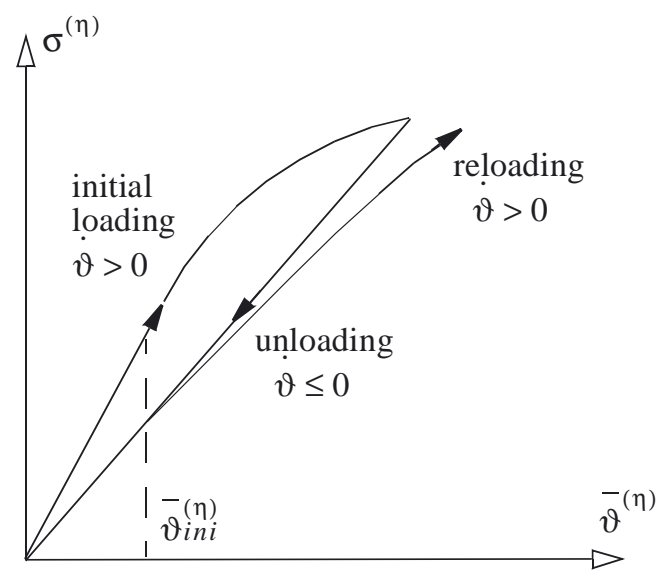

Fatigue Damage Cumulation

\section{FIGURE 2. Comparison Between Static and Fatigue Damage Cumulation Laws}

Based on the same concept, two similar fatigue damage cumulative laws have been developed in [35] for homogeneous material and [36] for concrete on macroscopic scale. It has been proved in [36], by assuming constant exponent in the power law, that fatigue damage cumulation law in the form of (33) can be reduced to the modified Palmgreen-Miner's model [16]. More sophisticated fatigue damage model reveals that the exponent is better off defined as a stress dependent parameter, i.e. the function of the maximum stress and mean stress value in uniaxial cyclic loading [10][35]. In this sense, we assume that parameter $\gamma^{(\eta)}$ depends on the mean and the maximum values of principal phase stresses, i.e.

$$
\gamma^{(\eta)}=g\left(c_{i}^{(\eta)}, \bar{\sigma}_{\text {max }}^{(\eta)}, \bar{\sigma}_{\text {mean }}^{(\eta)}\right)
$$

where $c_{i}^{(\eta)}$ is a set of material constants; $\bar{\sigma}_{\text {max }}^{(\eta)}$ and $\bar{\sigma}_{\text {mean }}^{(\eta)}$ are dimensionless quantities defined as

$$
\bar{\sigma}_{\text {max }}^{(\eta)}=\frac{\hat{\sigma}_{1 \max }^{(\eta)}}{2 \sigma_{u}^{(\eta)}} \quad \text { and } \quad \bar{\sigma}_{\text {mean }}^{(\eta)}=\frac{\hat{\sigma}_{1 \max }^{(\eta)}+\hat{\sigma}_{1 \min }^{(\eta)}}{2 \sigma_{u}^{(\eta)}}
$$

where $\hat{\sigma}_{1 \text { max }}^{(\eta)}$ and $\hat{\sigma}_{1 \text { min }}^{(\eta)}$ are the maximal and minimal principal phase average stresses at a given global position; $\sigma_{u}^{(\eta)}$ is the ultimate strength of the phase material. The calibration of the material constants in (34) is not trivial, since $\gamma^{(\eta)}$ is a function of the phase average stresses, whereas the only experimental data available is the number of cycles to failure, or fatigue life $N_{c r}$. Thus to calibrate material constants we set the following inverse problem: Find the material constants, $c_{i}^{(\eta)}$, so that $\left\|N_{c r}^{*}\left(c_{i}^{(\eta)}\right)-N_{c r}\right\|_{2}$ is minimal, where $N_{c r}=\left[N_{c r}^{1}, \ldots, N_{c r}^{k}\right]^{T}$ is a set of experimental fatigue life predictions obtained with various cyclic 
loading conditions; $N_{c r}^{*}\left(c_{i}^{(\eta)}\right)$ is a set of predicted fatigue life. The Jacobian matrix for the least square analysis could be evaluated using the finite difference method.

Another important issue in modeling the fatigue damage cumulation is the different effect of tension and compression. In fatigue process, it is well recognized that the damage growth and stop are not strictly in accordance with the tension and compression [15][31][41]. We notice that the definition of damage "driving force" in (27) can not account for such kind of phenomena. To remedy this incapability, we redefine the damage equivalent strain $\vartheta^{(\eta)}$ as follows:

$$
\bar{\vartheta}^{(\eta)}=\sqrt{\frac{1}{2} \hat{L}_{i j k l}^{(\eta)} \hat{\varepsilon}_{m n}^{(\eta)} F_{i j m n}^{(\eta)} \hat{\varepsilon}_{s t}^{(\eta)} F_{k l s t}^{(\eta)}}
$$

where $\hat{L}_{i j k l}^{(\eta)}$ and $\hat{\varepsilon}_{i j}^{(\eta)}$ represent the elastic phase constitutive tensor and nonlocal phase strain tensor, respectively, both expressed in principal directions; $F_{i j k l}^{(\eta)}$ denotes the phase strain weighting tensor. In matrix notation equation (36) is given as

$$
\bar{\vartheta}^{(\eta)}=\sqrt{\frac{1}{2}\left(\boldsymbol{F}^{(\eta)} \hat{\varepsilon}^{(\eta)}\right)^{T} \hat{\boldsymbol{L}}^{(\eta)}\left(\boldsymbol{F}^{(\eta)} \hat{\varepsilon}^{(\eta)}\right)}
$$

where the superscript $T$ represents the transpose; the principal nonlocal phase strain can be written as $\hat{\varepsilon}^{(\eta)}=\left[\varepsilon_{1}^{(\eta)}, \varepsilon_{2}^{(\eta)}, \varepsilon_{3}^{(\eta)}\right]^{T}$ and the phase strain weighting matrix is defined as

$$
\boldsymbol{F}^{(\eta)}=\left[\begin{array}{ccc}
h_{1}^{(\eta)} & 0 & 0 \\
0 & h_{2}^{(\eta)} & 0 \\
0 & 0 & h_{3}^{(\eta)}
\end{array}\right]
$$

where $h_{\xi}^{(\eta)} \equiv h\left(\varepsilon_{\xi}^{(\eta)}\right), \quad \xi=1,2,3$ are the weighting functions for each component of principal strain. The definition of weighting function $h\left(\varepsilon_{\xi}^{(\eta)}\right)$ is dependent of material properties and environmental conditions. The detail exploration on the effect of tension and compression on fatigue damage cumulation prior to the macro crack initiation is out of the content of this paper. We refer the interested readers to [41] for the comprehensive discussion. Here, we merely introduce a heuristic expression in the form of

$$
h_{\xi}^{(\eta)} \equiv h\left(\varepsilon_{\xi}^{(\eta)}\right)=\frac{1}{2}+\frac{1}{\pi} \operatorname{atan}\left[a_{1}\left(\varepsilon_{\xi}^{(\eta)}-a_{2}\right)\right], \quad \xi=1,2,3
$$

where the constants $a_{1}$ and $a_{2}$ are schematically illustrated in Figure 3. In the limit, as $a_{1} \rightarrow \infty$ and $a_{2}=0$, the weight function reduces to $h\left(\varepsilon_{\xi}^{(\eta)}\right)=\left\langle\varepsilon_{\xi}^{(\eta)}\right\rangle_{+} / \varepsilon_{\xi}^{(\eta)}$, which corresponds to the case where phase strain in compression does not promote damage. 


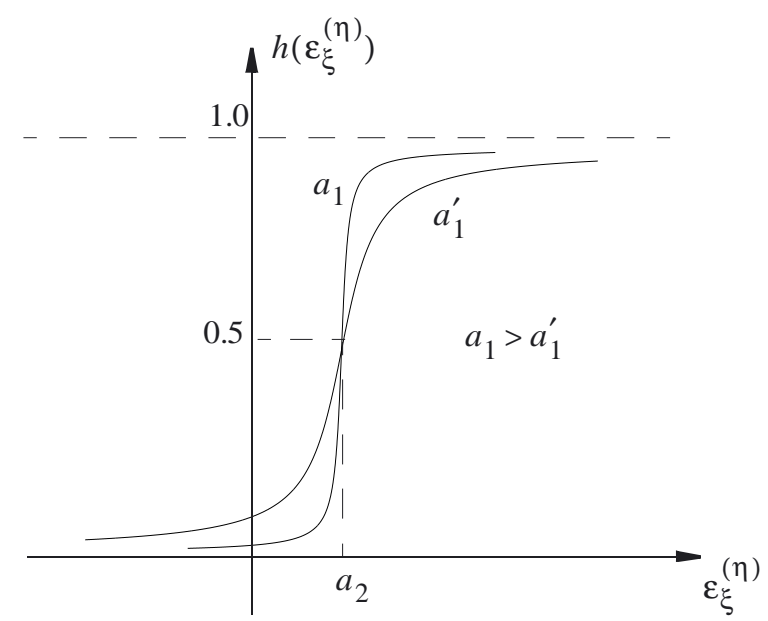

FIGURE 3. Phase Strain Weighting Function

\subsection{Computational issues}

In this section, we focus on computational aspects of the CDM based multiscale fatigue model developed in the previous two sections. We assume that the composite material consists of two phases, matrix $(\eta=m)$ and reinforcement $(\eta=f)$, denoted by $\Theta^{(m)}$ and $\Theta^{(f)}$ such that $\Theta=\Theta^{(m)} \cup \Theta^{(f)}$. For simplicity, we assume that fatigue damage occurs in the matrix phase only, i.e. $\omega^{(f)} \equiv 0$. The volume fractions for matrix and reinforcement are denoted as $v^{(m)}$ and $v^{(f)}$, respectively, such that $v^{(m)}+v^{(f)}=1$. The overall elastic properties (17) are given as

$$
\bar{L}_{i j k l}=v^{(m)} L_{i j m n}^{(m)} A_{m n k l}^{(m)}+v^{(f)} L_{i j m n}^{(f)} A_{m n k l}^{(f)}
$$

and the overall stress defined in (21) reduces to

$$
\bar{\sigma}_{i j}=v^{(m)} \sigma_{i j}^{(m)}+v^{(f)} \sigma_{i j}^{(f)}
$$

where the nonlocal phase average stresses $\sigma_{i j}^{(m)}$ and $\sigma_{i j}^{(f)}$ are defined by (20). Due to nonlinear character of the problem an incremental finite element analysis is employed. Prior to nonlinear analysis elastic strain concentration factors, $A_{i j k l}(\boldsymbol{y})$, are precomputed using (12) and (11) in the microscopic domain (RVE) by either finite element method or, if possible, by solving an inclusion problem analytically. Subsequently, the phase elastic strain concentration factors $A_{i j k l}^{(\eta)}(\eta=m, f)$ and damage strain concentration factors $G_{i j k l}^{(\eta)}$ are precomputed using (19).

In the remaining of this section we focus on three computational issues: (i) implicit micro and macro stress update (integration) procedures, (ii) consistent linearization, and (iii) integration of the phase fatigue damage cumulative law. 


\subsection{Stress Update Procedures}

Given: the displacement vector ${ }_{t} u_{m}$; the overall strain ${ }_{t} \bar{\varepsilon}_{m n}$; the damage variable ${ }_{t} \omega^{(m)}$; the displacement increment $\Delta u_{m}$ calculated from the incremental finite element analysis of the macro problem. The left subscript denotes the increment step, i.e., $t+\Delta t \square \quad$ is the variables at the current increment, whereas ${ }_{t} \square \quad$ is a converged variable from the previous increment. For simplicity, we will often omit the left subscript for the current increment, i.e., $\square \equiv{ }_{t+\Delta t} \square$.

Find: the displacement vector $u_{m} \equiv{ }_{t+\Delta t} u_{m}={ }_{t} u_{m}+\Delta u_{m}$; the overall strain $\bar{\varepsilon}_{m n}$; the nonlocal phase strains $\varepsilon_{m n}^{(m)}$ and $\varepsilon_{m n}^{(f)}$; the nonlocal phase damage variable $\omega^{(m)}$; the overall stress $\bar{\sigma}_{m n}$ and the nonlocal phase stresses $\sigma_{m n}^{(m)}$ and $\sigma_{m n}^{(f)}$.

The stress update procedure consists of the following steps:

i.) Calculate the macroscopic strain increment, $\Delta \bar{\varepsilon}_{m n}=\Delta u_{\left(m, x_{n}\right)}$ and update the macroscopic strains by $\bar{\varepsilon}_{m n}={ }_{t} \bar{\varepsilon}_{m n}+\Delta \bar{\varepsilon}_{m n}$.

ii.) Compute the principal components of $\varepsilon_{m n}^{(m)}$ by (18) and the damage equivalent strain $\bar{\vartheta}^{(m)}$ by (37) in terms of ${ }_{t} \omega^{(m)}$ and $\bar{\varepsilon}_{m n}$.

iii.) Check the inelastic/elastic process conditions by (33) where $\dot{\bar{\vartheta}}^{(m)}$ is integrated as $t+\Delta t \Delta \bar{\vartheta}^{(m)}=\bar{\vartheta}^{(m)}-{ }_{t} \bar{\vartheta}^{(m)}$

$$
\text { If inelastic process, i.e. } \bar{\vartheta}^{(m)}>\bar{\vartheta}^{(m)} \text { and } \bar{\vartheta}^{(m)} \geq \bar{\vartheta}_{\text {ini }}^{(m)} \text {, then update } \omega^{(m)} \text { through integration of (33). }
$$

The integration of the fatigue damage cumulative law (33) is carried out using the backward Euler scheme such that

$$
\boldsymbol{\aleph}^{(m)} \equiv \omega^{(m)}-{ }_{t} \omega^{(m)}-{ }_{t+\Delta t}\left[\frac{\Phi^{(m)}}{\omega^{(m)}}\right]^{\gamma^{(m)}}\left[\frac{\partial \Phi^{(m)}}{\partial \bar{\vartheta}^{(m)}}\right]\left(\bar{\vartheta}^{(m)}-\bar{\vartheta}^{(m)}\right)=0
$$

Since $\bar{\vartheta}^{(m)}$ is governed by the current average strains in the matrix phase which in turn depend on the current damage variable, it follows that (42) is a nonlinear function of $\omega^{(m)}$. Newton method is used to solve for $\omega^{(m)}$ :

$$
{ }^{i+1} \omega^{(m)}={ }^{i} \omega^{(m)}-\left.\left[\left(\frac{\partial \boldsymbol{\aleph}^{(m)}}{\partial \omega^{(m)}}\right)^{-1} \boldsymbol{\aleph}^{(m)}\right]\right|_{{ }^{(m} \omega^{(m)}}
$$

where the derivation of the derivative term in (43) is given in the Appendix.

Otherwise, for elastic process: $\omega^{(m)}={ }_{t} \omega^{(m)}$.

vi.) Update the nonlocal phase strains $\varepsilon_{m n}^{(m)}$ and $\varepsilon_{m n}^{(f)}$ by (18). 
v.) Calculate the nonlocal phase stresses $\sigma_{i j}^{(m)}$ and $\sigma_{i j}^{(f)}$ using (20) and update the macroscopic stresses $\bar{\sigma}_{i j}$ defined by (41).

\subsection{Consistent Tangent Stiffness}

In this subsection, we focus on the derivation of the consistent tangent stiffness matrix for the macro-problem. We start by substituting (18) into (20) and then take material derivative of the incremental form of (20) in the matrix domain $(\eta=m)$, which yields

$$
\dot{\sigma}_{i j}^{(m)}=P_{i j m n}^{(m)} \dot{\bar{\varepsilon}}_{m n}+Q_{i j m n}^{(m)} \bar{\varepsilon}_{m n} \dot{\omega}^{(m)}
$$

where

$$
\begin{gathered}
P_{i j m n}^{(m)}=\left(1-\omega^{(m)}\right) L_{i j k l}^{(m)}\left(A_{k l m n}^{(m)}+G_{k l s t}^{(m)} D_{s t m n}^{(m)}\right) \\
Q_{i j m n}^{(m)}=\left(1-\omega^{(m)}\right) L_{i j k l}^{(m)} G_{k l s t}^{(m)} R_{s t m n}^{(m)}-L_{i j k l}^{(m)}\left(A_{k l m n}^{(m)}+G_{k l s t}^{(m)} D_{s t m n}^{(m)}\right)
\end{gathered}
$$

The fourth order tensor $R_{s t m n}^{(m)}$ is obtained by taking derivative of the nonlocal matrix strain $\varepsilon_{k l}^{(m)}$ defined in (18) with respect to $\omega^{(m)}$ such that

$$
\begin{gathered}
\frac{\partial \varepsilon_{k l}^{(m)}}{\partial \omega^{(m)}}=G_{k l s t}^{(m)} R_{s t m n}^{(m)} \bar{\varepsilon}_{m n} \\
R_{s t m n}^{(m)}=\left(I_{s t p q}-B_{s t p q}^{(m)} \omega^{(m)}\right)^{-2} C_{p q m n}^{(m)}
\end{gathered}
$$

To obtain $\dot{\omega}^{(m)}$, we make use of damage cumulative law (33) with the inelastic/elastic process conditions defined in Section 3. In the case of elastic process, we have $\dot{\omega}^{(m)}=0$. For inelastic process, the derivation of $\dot{\omega}^{(m)}$ is detailed in the Appendix and the final result can be expressed as

$$
\dot{\omega}^{(m)}=W_{i j}^{(m)} \dot{\bar{\varepsilon}}_{i j}
$$

Substituting (49) into (44) and manipulating the indices, we get the following relation between the rate of the overall strain and nonlocal phase stresses in the matrix domain

$$
\dot{\sigma}_{i j}^{(m)}=\wp_{i j m n}^{(m)} \dot{\bar{\varepsilon}}_{m n}
$$

where

$$
\wp_{i j m n}^{(m)}=P_{i j m n}^{(m)}+Q_{i j s t}^{(m)} \bar{\varepsilon}_{s t} W_{m n}^{(m)}
$$


A similar result relating the rate of the nonlocal reinforcement stress and the overall strain rate can be obtained by substituting (18) into (20) and then taking material derivative of (20) in the reinforcement domain $(\eta=f)$

$$
\dot{\sigma}_{i j}^{(f)}=\wp_{i j m n}^{(f)} \dot{\bar{\varepsilon}}_{m n}
$$

where

$$
\begin{gathered}
\wp_{i j m n}^{(f)}=P_{i j m n}^{(f)}+Q_{i j s t}^{(f)} \bar{\varepsilon}_{s t} W_{m n}^{(m)} \\
P_{i j m n}^{(f)}=L_{i j k l}^{(f)}\left(A_{k l m n}^{(f)}+G_{k l s t}^{(f)} D_{s t m n}^{(m)}\right) \\
Q_{i j s t}^{(f)}=L_{i j k l}^{(f)} G_{k l m n}^{(f)} R_{m n s t}^{(m)}
\end{gathered}
$$

Finally, the overall consistent tangent stiffness is constructed by substituting (50) and (52) into the rate form of the overall stress-strain relation (41)

$$
\begin{gathered}
\dot{\bar{\sigma}}_{i j}=\wp_{i j m n} \dot{\bar{\varepsilon}}_{m n} \\
\wp_{i j m n}=v^{(m)} \wp_{i j m n}^{(m)}+v^{(f)} \wp_{i j m n}^{(f)}
\end{gathered}
$$

\subsection{Integration of Fatigue Damage Cumulative Law}

To develop an efficient accelerating technique for the integration of fatigue damage cumulative law, a constant amplitude cyclic loading history is typically subdivided into a series of load cycle blocks, and each block consists of several load cycles. One of the integration schemes developed in [36] assumes that in each block of cycles, the mechanical response is independent of fatigue damage cumulation until the local rupture occurs. In another model developed for homogeneous materials [12], the first cycle in the block in which the damage increment is caused by inelastic deformation, is used to compute a constant rate of fatigue damage growth in that block. The major shortcomings of this model are threefold: (i) the deviation from the equilibrium path caused by the integration of fatigue damage cumulation law, (ii) the difficulty in estimating an adequate block size, especially in the initial and near-rupture loading stages where the growth of fatigue damage is very rapid, and (iii) applicability to heterogeneous materials.

In what follows the damage cumulative law will be approximated by the first order initial value problem with respect to the number of load cycles, and subsequently solved using the adaptive modified Euler's method with the maximum damage increment control and consistency adjustment.

Let us return to the fatigue damage cumulative law defined in (33). Since this fatigue law is stated in the rate form, it is necessary to integrate it along the loading path to obtain the current damage state. The nonlocal matrix phase damage increment in one load cycle can be expressed as 


$$
\int_{t}^{\left(t+\tau_{0}\right)} \dot{\omega}^{(m)} d t=\int_{t}^{\left(t+\tau_{0}\right)}\left[\frac{\Phi^{(m)}}{\omega^{(m)}}\right]^{\gamma^{(m)}} \frac{\partial \Phi^{(m)}}{\partial \dot{\vartheta}^{-(m)}}<\dot{\vartheta}^{(m)}>_{+} d t
$$

where $t$ is the time at the beginning of a load cycle and $\tau_{0}$ is the period of the cyclic loading. The above integration has to be carried at each Gauss point in the macro domain. Assuming that the increment of phase damage in one load cycle is very small, we can approximate the derivative of the nonlocal damage parameter with respect to the number of load cycles as

$$
\left.\left.\frac{d \omega^{(m)}}{d N}\right|_{K} \approx \int_{t}^{\left(t+\tau_{0}\right)} \dot{\omega}^{(m)} d t \equiv \Delta \omega^{(m)}\right|_{K}=\left.\omega^{(m)}\right|_{K}-\left.\omega^{(m)}\right|_{K-1}
$$

where $N$ denotes the number of load cycles; $\left.\omega^{(m)}\right|_{K}$ is the phase damage at the end of load cycle $K$ which can be obtained by the incremental finite element analysis for this cycle with initial damage $\left.\omega^{(m)}\right|_{K-1}$ and the corresponding initial strain/stress conditions.

Using the forward Euler's method the nonlocal phase damage after $\Delta N_{K}$ cycles from the current load cycle $K$ can be approximated by

$$
\left.\omega^{(m)}\right|_{\left(K+\Delta N_{K} ; \Delta N_{K}\right)}=\left.\omega^{(m)}\right|_{K}+\left.\Delta N_{K} \Delta \omega^{(m)}\right|_{K}
$$

where $\left.\omega^{(m)}\right|_{\left(K+\Delta N_{K} ; \Delta N_{K}\right)}$ represents the approximate solution of the nonlocal phase damage at the end of load cycle $K+\Delta N_{K}$ with the block size $\Delta N_{K}$ and the initial nonlocal damage $\left.\omega^{(m)}\right|_{K}$.

It is important to note that updating the damage variable while keeping the rest of the fields fixed violates constitutive equations. This inconsistency is subsequently alleviated in two steps: (i) update the nonlocal phase stresses using the overall strain from the end of cycle $K,\left.\bar{\varepsilon}_{i j}\right|_{K}$, (ii) carry out nonlinear finite element analysis to equilibrate discrete equilibrium equations. We will refer to this two-step process as the consistency adjustment.

For forward Euler's one-step method the block size $\Delta N_{K}$ should be selected to ensure accuracy. The block size can be adaptively selected by keeping the nonlocal phase damage increment sufficiently small when the damage increases rapidly and vice versa. This can be expressed as follows:

$$
\left.\Delta N_{K}=\operatorname{int}\left\{\Delta \omega_{a}^{(m)} /\left.\max _{\text {gauss }}\left(\Delta \omega^{(m)}\right)\right|_{K}\right)\right\}
$$

where operator $\operatorname{int}\{\bullet\}$ denotes the truncation to the decimal part; $\Delta \omega_{a}^{(m)}$ is a user-defined allowable tolerance of phase damage increment per cycle; $\max (\quad)$ is computed with respect to all integration points in the macro-problem. There are two major reasons to monitor the value of maximum damage increment. First, is to ensure the existence of the initial value problem, i.e., if the damage growth rate in cycle $K$ in at least one of the Gauss points is very high, the approximation of the initial value problem might be inaccu- 
rate, and thus the block size $\Delta N_{K}$ evaluated by (61) should be set to zero. In this case, the method reduces to the direct cycle-by-cycle approach. The second reason is to ensure accuracy of the aforementioned consistency adjustment process.

The fatigue life, denoted as $N_{\max }$, can be expressed as

$$
N_{\text {max }}=n+\sum_{K=1}^{n} \Delta N_{K} ; \quad \Delta N_{K} \geq 0
$$

where $n$ is the number of the cycle blocks in the loading history which is also the actual number of the cycles carried out in the case of the direct simulation. The maximal value of $n$ is $\operatorname{int}\left\{1 /\left(\Delta \omega_{a}^{(m)}\right)\right\}$ provided that the failure occurs when $\omega^{(m)}$ reaches one at the critical Gauss point.

To control solution accuracy of the initial value problem we adopt the modified Euler's integrator [40] with the initial block size determined by (61). The nonlocal phase damage at load cycle $K+\Delta N_{K}(60)$ is then defined as

$$
\left.\omega^{(m)}\right|_{\left(K+\Delta N_{K} ; \Delta N_{K}\right)}=\left.\omega^{(m)}\right|_{K}+\frac{\Delta N_{K}}{2}\left(\left.\Delta \omega^{(m)}\right|_{K}+\left.\Delta \omega^{(m)}\right|_{K+\Delta N_{K}}\right)
$$

where $\left.\Delta \omega^{(m)}\right|_{K}$ is evaluated by (59) while $\left.\Delta \omega^{(m)}\right|_{K+\Delta N_{K}}$ is also obtained by (59) after substituting $K+\Delta N_{K}$ for $K ;\left.\omega^{(m)}\right|_{K+\Delta N_{K}}$ is the first order approximation defined in (60).

The problem of integration of phase fatigue damage cumulative law can be stated as follows:

Given: the tolerance err; allowable damage increment per load cycle $\Delta \omega_{a}^{(m)}$; initial nonlocal phase damage in the matrix phase $\left.\omega^{(m)}\right|_{K-1}$, and the overall strain $\left.\bar{\varepsilon}_{m n}\right|_{K-1}$ at the beginning of load cycle $K$.

Find: the size of the block $\Delta N_{K}$; fatigue life $N_{\text {max }}$; nonlocal phase damage and overall strain at the end of cycle $K+\Delta N_{K}$.

The adaptive scheme is summarized as follows:

i.) Carry out the incremental finite element analysis, as described in Section 4.1 and 4.2, for one load cycle with initial nonlocal phase damage $\left.\omega^{(m)}\right|_{K-1}$ and the overall strain $\left.\bar{\varepsilon}_{m n}\right|_{K-1}$. Denote the nonlocal phase damage at the end of this cycle as $\left.\omega^{(m)}\right|_{K}$, and the overall strain as $\left.\bar{\varepsilon}_{m n}\right|_{K}$. At each integration point in the macro domain estimate the rate of nonlocal phase damage in the current load cycle, $\left.\Delta \omega^{(m)}\right|_{K}$, as defined by (59).

ii.) Calculate the initial block size using (61). 
iii.) At each integration point in the macro domain, compute the approximate solution $\left.\omega^{(m)}\right|_{\left(K+\Delta N_{K} ; \Delta N_{K}\right)}$ with the block size $\Delta N_{K}$ using the modified Euler's method (63); then using the block size $\Delta N_{K} / 2$, compute $\left.\omega^{(m)}\right|_{\left(K+\Delta N_{K} ; \Delta N_{K} / 2\right)}$ by two successive uses of (63).

vi.) Find the maximum error among all the integration points and check the convergence by:

$$
\underset{\text { gauss }}{\max }\left\{\left|\omega^{(m)}\right|_{\left(K+\Delta N_{K} ; \Delta N_{K}\right)}-\left.\omega^{(m)}\right|_{\left(K+\Delta N_{K} ; \Delta N_{K} / 2\right)} \mid\right\} \leq e r r
$$

If (64) is false, set $\Delta N_{K} \leftarrow \Delta N_{K} / 2$ and go back to iii). Otherwise, update the fatigue life by (62), i.e. $\left.N_{\max }\right|_{K}=\left.N_{\max }\right|_{K-1}+\Delta N_{K}+1$; update the approximation of the nonlocal phase damage at the end of load cycle $K+\Delta N_{K},\left.\omega^{(m)}\right|_{\left(K+\Delta N_{K} ; \Delta N_{K} / 2\right)}$, and the overall strain $\left.\bar{\varepsilon}_{m n}\right|_{K}$. Then, compute the nonlocal phase strains $\left.\varepsilon_{m n}^{(m)}\right|_{K}$ and $\left.\varepsilon_{m n}^{(f)}\right|_{K}$ using (18).

vii.) Perform consistency adjustment: (i) calculate nonlocal phase stresses $\left.\sigma_{i j}^{(m)}\right|_{K}$ and $\left.\sigma_{i j}^{(f)}\right|_{K}$ by (20), and macroscopic stresses $\left.\bar{\sigma}_{i j}\right|_{K}$ by (41); (ii) equilibrate discrete solution using nonlinear finite analysis. Finally, set $K \leftarrow K+1$ and go to $i$.) for the next block of cycles.

\subsection{Numerical Examples}

\subsection{Qualitative Examples for Two-Phase Fibrous Composites}

The first set of numerical examples investigates the computational efficiency and accuracy of the proposed fatigue model. We consider the classical stress concentration problem - a thin plate with a centered small circular hole, as shown in Figure 4. The plate is assumed to be composed of $0 / 0$ ply of fibrous composite. The plate is subjected to uniaxial tension perpendicular to the fiber direction. The fiber direction is aligned along the $\mathrm{Z}$ axis whereas the two transverse directions coincide with the $\mathrm{X}$ and $\mathrm{Y}$ axes. The properties of the two micro-phases are as follows:

Matrix: $v^{(m)}=0.733, E^{(m)}=69 \mathrm{GPa}, \mu^{(m)}=0.33$

Fiber: $\quad v^{(f)}=0.267, E^{(f)}=379 \mathrm{GPa}, \mu^{(f)}=0.21$

where $E, \mu$ and $G$ denote Young's modulus, Poisson ratio, and shear modulus, respectively. The parameters of the damage evolution law are chosen as $\alpha^{(m)}=8.2, \beta^{(m)}=10.2$ and $\vartheta_{0}=0.05(\mathrm{MPa})^{1 / 2}$. For simplicity, $\gamma^{(m)}$ is assumed to be constant, and set $\gamma^{(m)}=4.5$ for low-cycle fatigue, and $\gamma^{(m)}=15$ for high-cycle fatigue. 


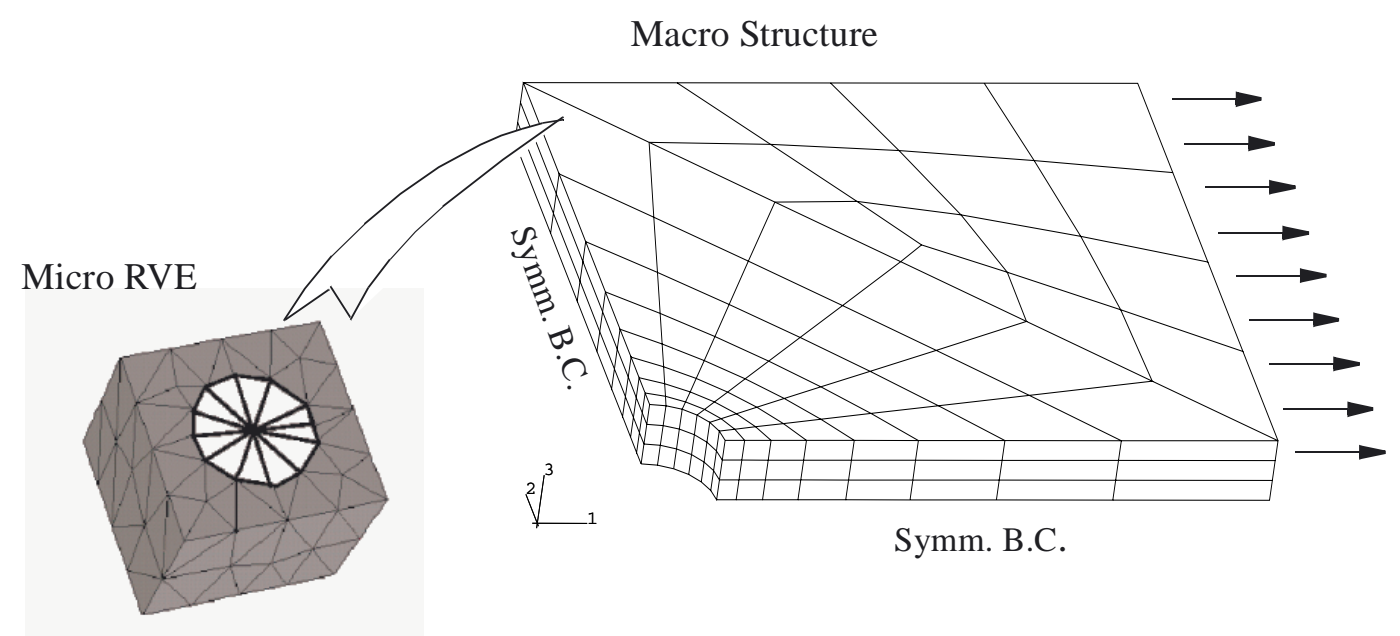

FIGURE 4. FE model of RVE and Macro Domain

The static loading capacity of the plate is $103.6 \mathrm{~N}$ as shown in Figure 5.

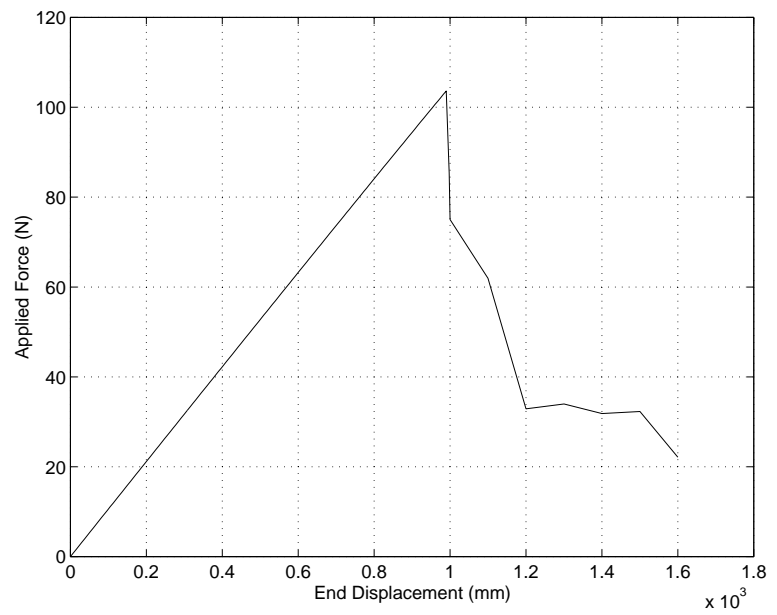

FIGURE 5. Static Loading Capacity

The cyclic loading is designed as a tension-to-zero loading with amplitude of $90 \mathrm{~N}$. The evolution of fatigue damage cumulation and the nonlocal matrix strain at the critical point are illustrated in Figures 6-8. 

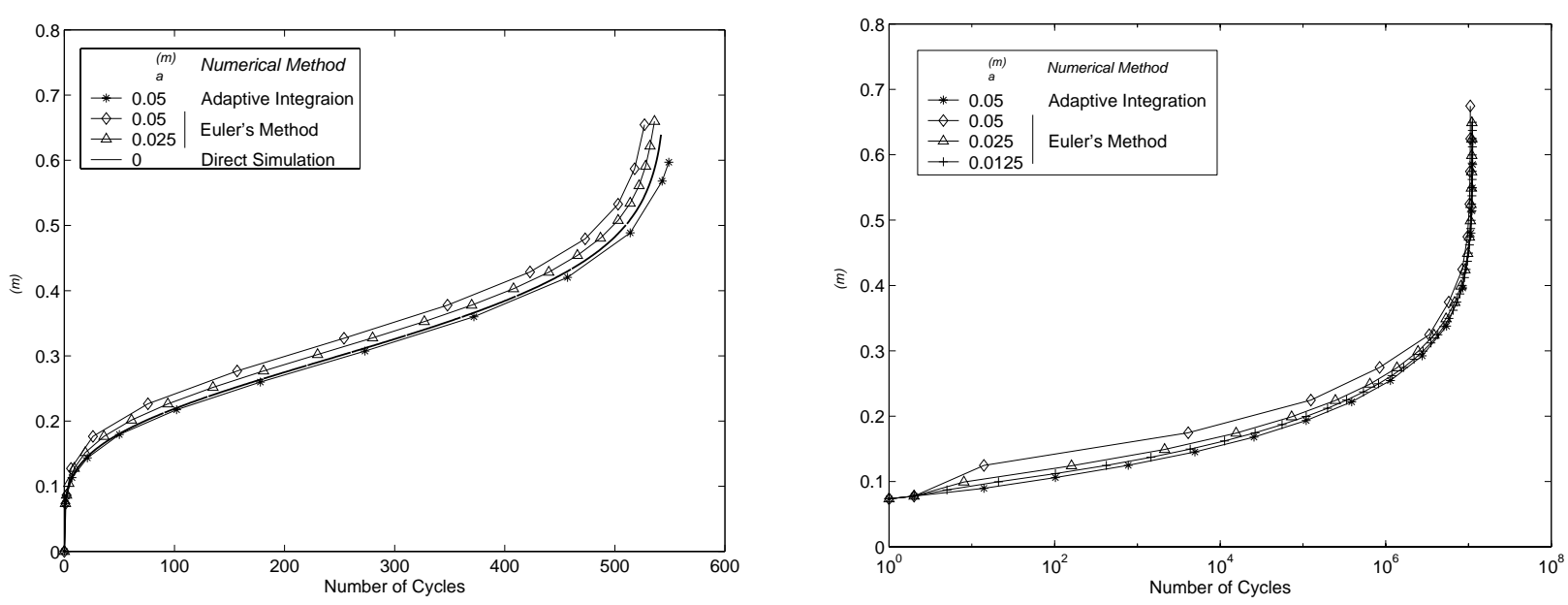

FIGURE 6. Fatigue Damage Cumulation for Low-Cycle and High-Cycle Fatigue
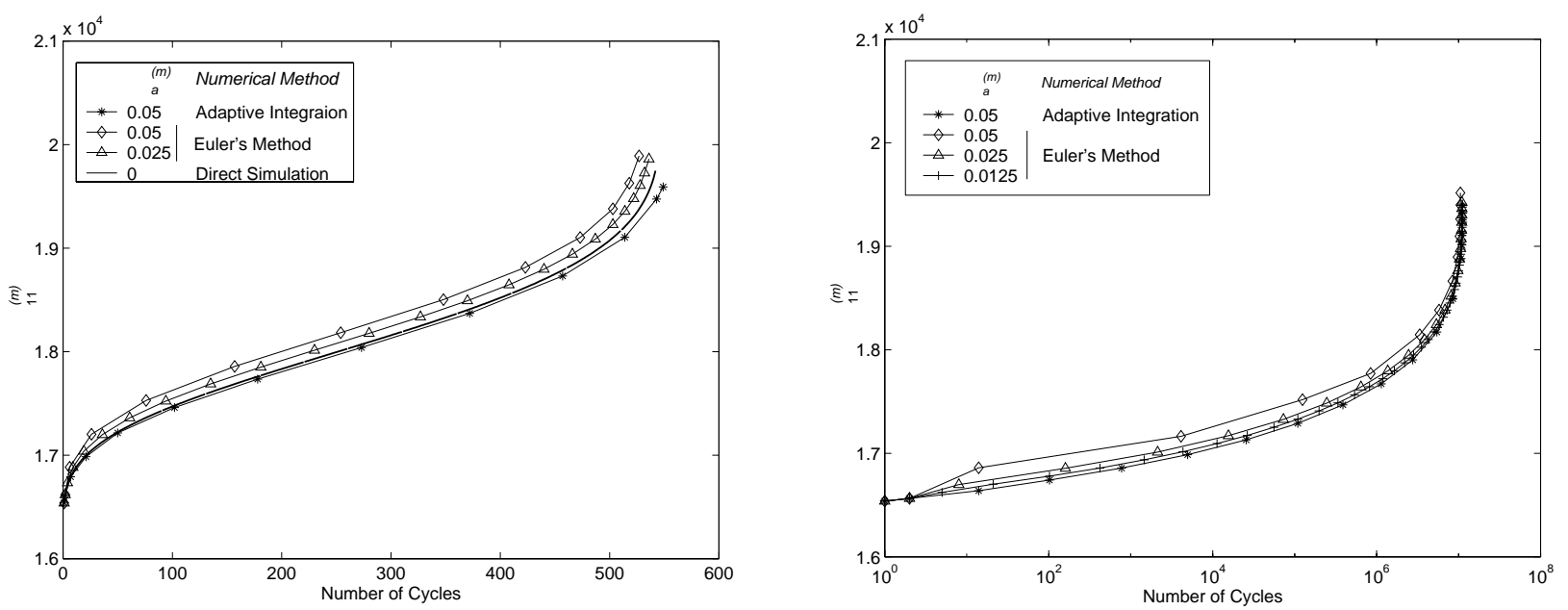

FIGURE 7. Strain Softening for Low-Cycle and High-Cycle Fatigue 

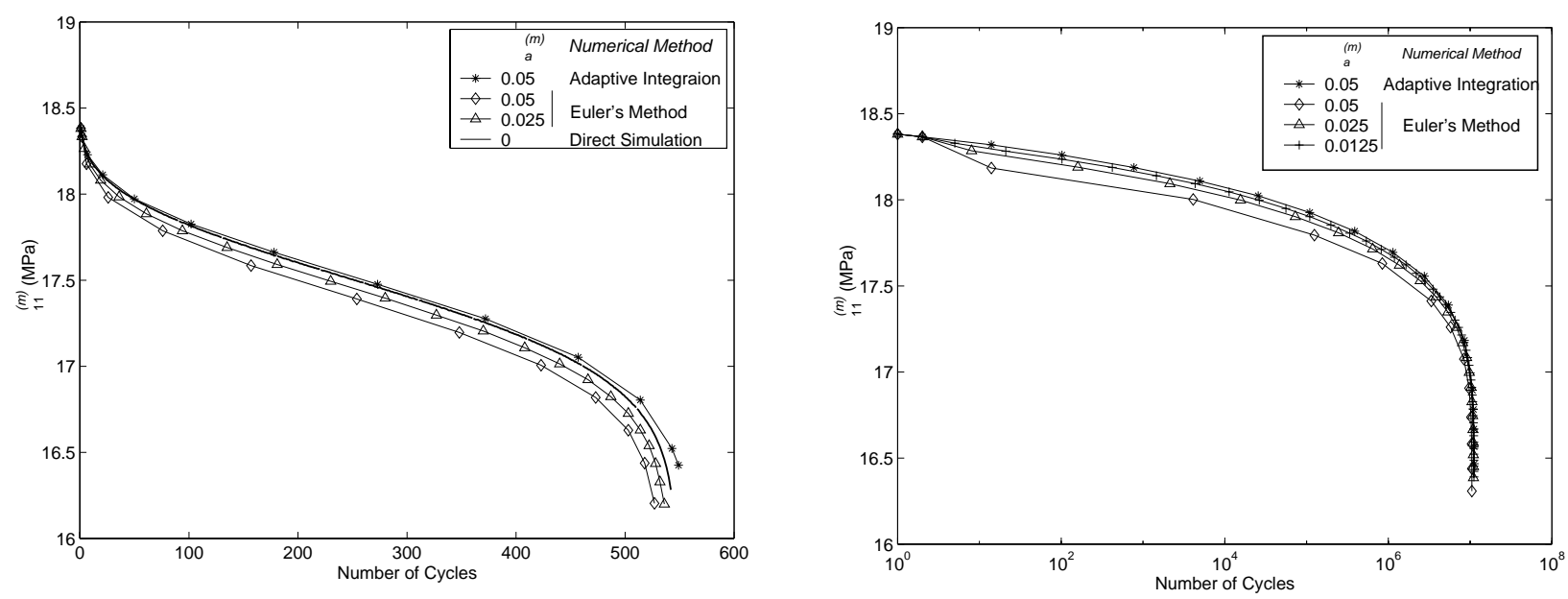

FIGURE 8. Local Stress Relaxation for Low-Cycle and High-Cycle Fatigue

For low-cycle fatigue, the direct cycle-by-cycle simulation serves as a reference solution. Several allowable damage increments per cycle were selected to study the convergence of the method. Indeed, the results summarized in Figures 6-8 demonstrate excellent convergence characteristics of the proposed fatigue model. For high-cycle fatigue problems the solution obtained by the forward Euler method with very small $\Delta \omega_{a}^{(m)}$ is used as a reference solution instead of the direct simulation which is computationally prohibitive. Similar observations can be made for high-cycle problem.

\subsection{Large Scale Fatigue Analysis For Woven Composites}

In this section, we consider the tailcone exhaust structure made of Techniweave T-Form Nextel312/Blackglas Composite System as shown in Figure 9. The fabric designs used 600 denier bundles of Nextel 312 fibers surrounded by Blackglas 493C matrix material [8]. The bundles are assumed to be linear elastic (damage-free) throughout the analysis.
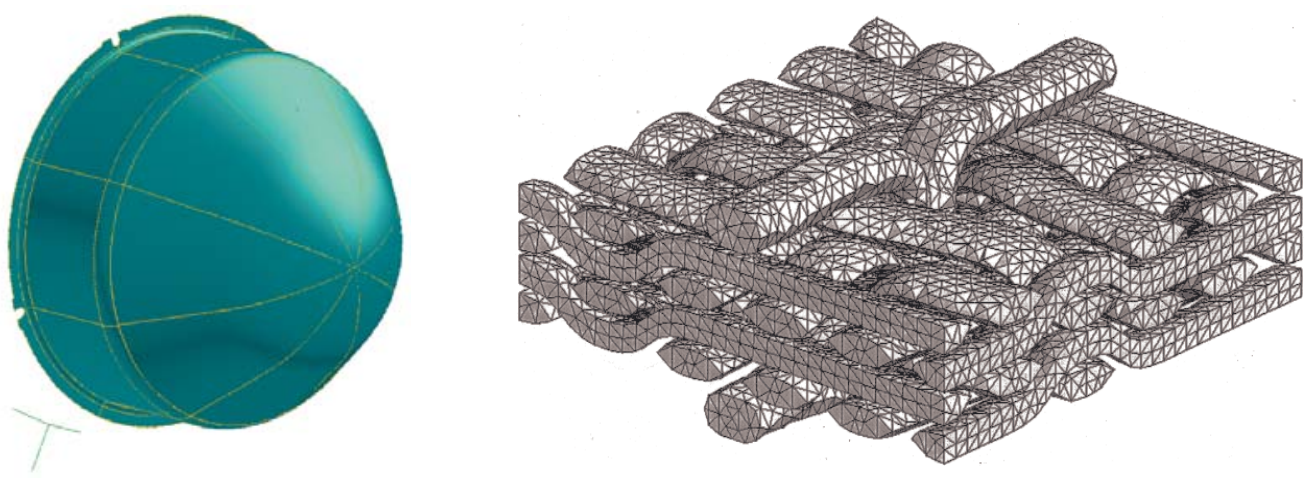

FIGURE 9. Geometric Model of the Techniweave T-Form Woven Microstructure 
Here, we assume the woven composite to be a periodic two-phase material composed of bundles and matrix. The phase properties of RVE are summarized below:

Blackglas Matrix: $v^{(m)}=0.565, E^{(m)}=38.61 \mathrm{GPa}, \mu^{(m)}=0.26$

Bundle: $v^{(f)}=0.435, E_{A}^{(f)}=114.28 \mathrm{GPa}, G_{A}^{(f)}=45.19 \mathrm{GPa}, \mu_{A}^{(f)}=0.244$,

$$
E_{T}^{(f)}=112.10 \mathrm{GPa}, G_{T}^{(f)}=44.95 \mathrm{GPa}
$$

where the subscripts $A$ and $T$ represent the axial and transverse directions for transversely isotropic material.

The microstructure of RVE is discretized with 20,558 nodes and 98,282 elements totaling 61,650 degrees of freedom as shown in Figure 9, where the matrix phase has been removed in order to give a clear view of boundles. The compressive principal strains have been observed to have little effect on the damage cumulation, so the constants in (38) are selected as $a_{1}=10^{7}$ and $a_{2}=0$. The material constants $\alpha^{(m)}, \beta^{(m)}$ and $\vartheta_{0}$ in (32) have been calibrated based on the tensile test under quasi-static uniaxial loading in the weave plane [8], which gives $\alpha^{(m)}=7.6, \beta^{(m)}=10.9$ and $\vartheta_{0}^{(m)}=0.24(\mathrm{MPa})^{1 / 2}$. The endurance limit is taken as $\widehat{\vartheta}_{i n i}=0$. The predicted ultimate strength in weave plane was $105.8 \mathrm{MPa}$ with $0.178 \%$ ultimate strain. In the direction normal to the weave plane, the ultimate strength is $69.1 \mathrm{MPa}$ and the ultimate strain $0.21 \%$. Material constants were selected so that numerical results at ultimate points were in good agreement with the test data.

Following the procedure described in Section 3, we assumed that the fatigue parameter $\gamma^{(m)}(34)$ is in the form of

$$
\gamma^{(m)}=\left(\bar{\sigma}_{\text {max }}^{(m)}\right)^{c_{1}}\left\{c_{2}+c_{3}\left(\bar{\sigma}_{\text {max }}^{(m)}-\bar{\sigma}_{\text {mean }}^{(m)}\right)+c_{4}\left(\bar{\sigma}_{\text {max }}^{(m)}-\bar{\sigma}_{\text {mean }}^{(m)}\right)^{2}\right\}
$$

where $c_{1}-c_{4}$ are material constants. The calibration of the material constants in (65) is performed for the uniform cyclic tension-tension loading in the weave plane. The minimal tensile loading is ten percent of the maximum value. 


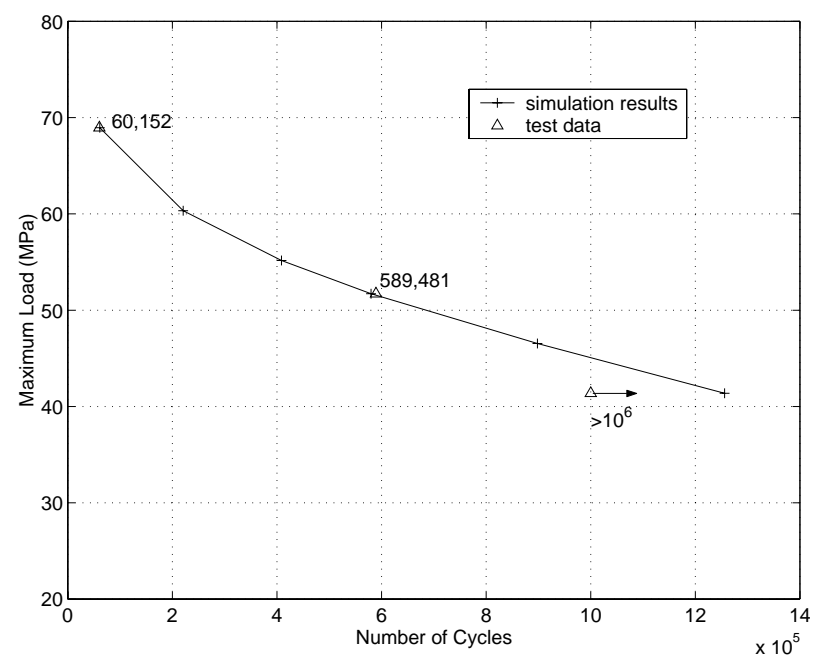

FIGURE 10. Predicted Fatigue Life and Test Data

Figure 10, compares the computed fatigue life with material constants $c_{1}=0.5, c_{2}=0.554, c_{3}=1.35$, $c_{4}=-2.68$ and test data. The ultimate strength of the matrix phase is $\sigma_{u}^{(m)}=69.1 \mathrm{MPa}$. Figure 11 illustrates the evolution of nonlocal matrix damage parameter and the nonlocal equivalent matrix stress.
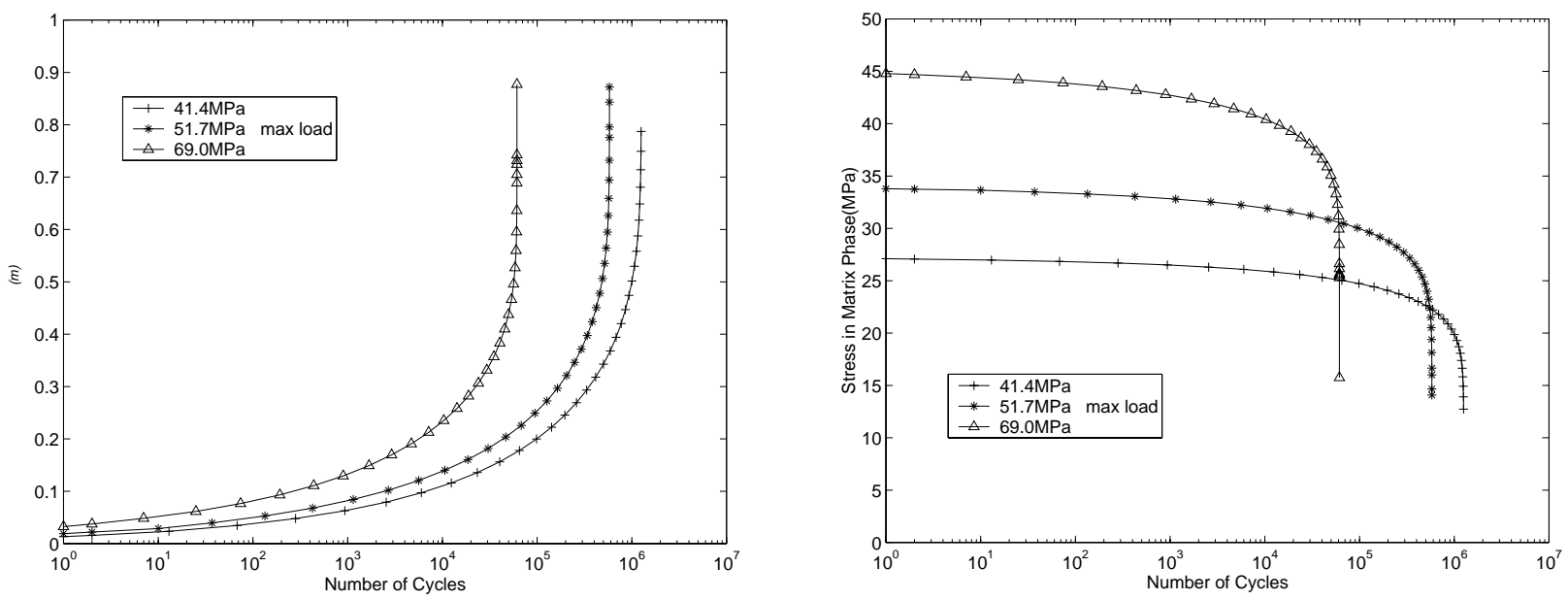

FIGURE 11. Damage Evolution and Stress Relaxation in Uniform Tension-Tension Cyclic Loading 

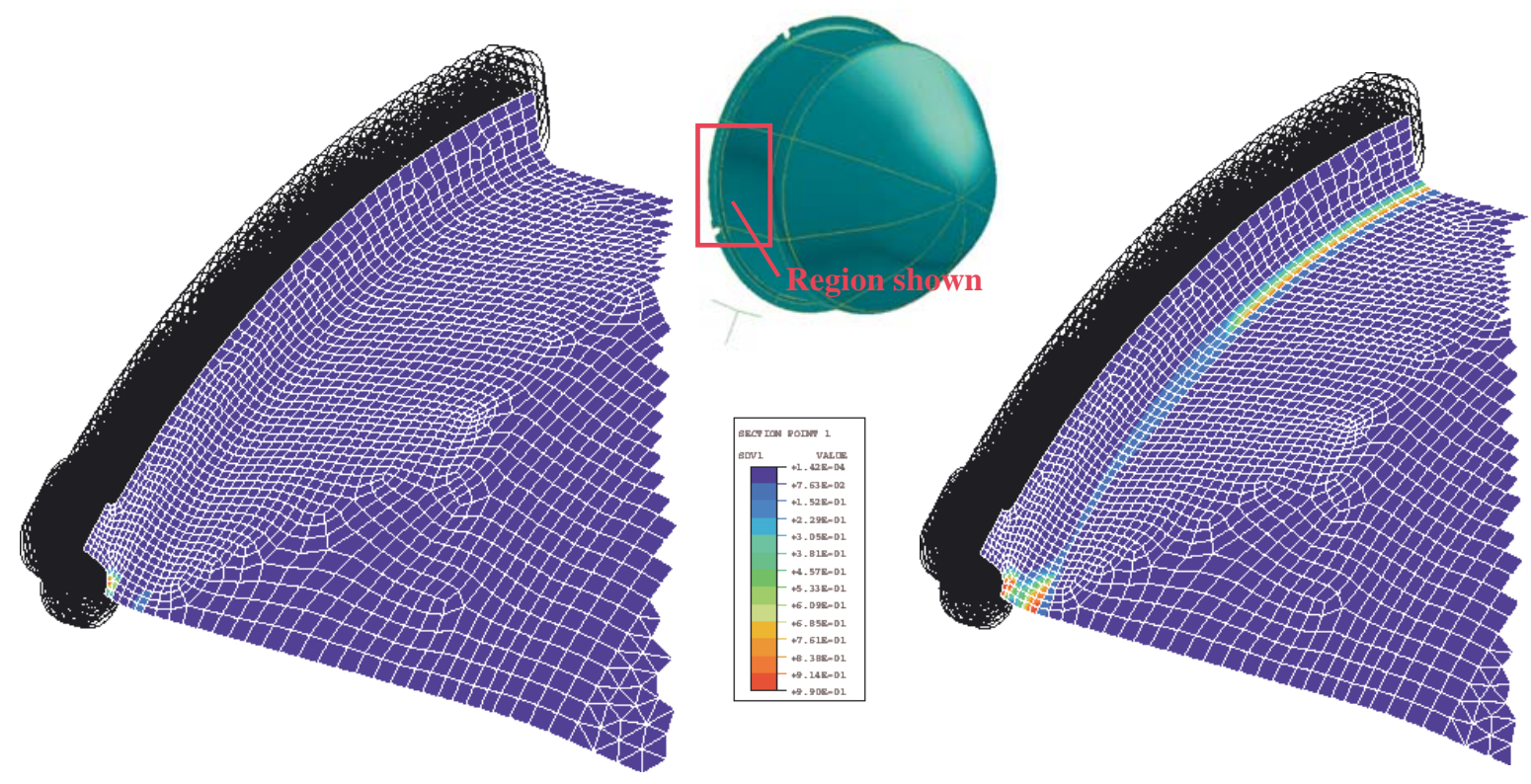

FIGURE 12. Damage Distribution in Critical Region of the Tailcone

Once the fatigue damage model has been calibrated, we turn to the evaluation of fatigue life of the exhaust tailcone structure of a aircraft engine. The finite element mesh of one-eighth of the tailcone model in the neighborhood of the attachment hole is shown in Figure 12. It consists of 3,154 nodes, 3,242 thin shell elements and 385 spring elements totaling 17,766 degrees of freedom. The cyclic internal pressure is applied to the shell structure with the minimum pressure being of the $10 \%$ of the maximum pressure. Since the model is the thin shell structure, membrane force is the dominant internal force which is approximately in the plane of weave. It can be seen that the damage initiates at the location of attachment and then quickly spreads around the supporting ring causing the overall structural failure. The critical state is reached after 1.12 million cycles. No experiments have been conducted up to failure to verify this result.

\subsection{Conclusions and Future Research Directions}

Traditionally, life predictions for macrocrack initiation are carried out using $\mathrm{S}-\mathrm{N}$ or $\varepsilon-\mathrm{N}$ curves in conjunction with some parameters designated to take into account the differences between actual components and test specimens such as geometry, fabrication, environmental conditions, etc. Due to the coupled nature of the present multiscale CDM based fatigue model, mechanical and fatigue damage cumulation analyses are carried out simultaneously without relying on $\mathrm{S}-\mathrm{N}$ or $\varepsilon-\mathrm{N}$ curves. The novel accelerating technique for the integration of CDM based fatigue damage cumulative law make it possible to simulate the damage growth by fully coupled finite element analysis for the real structure Thus large amount of specimen tests can be avoided, while complex geometrical features, material imperfections, multiaxial loading conditions, and material data can be readily incorporated into the computational model.

For macrocrack propagation problem, the present model has certain limitations. As observed in [22][23], the nonlocal damage theory may lead to the spurious damage zone widening phenomenon, especially when 
the crack opening is accompanied with large strains. Physically, with the evolution of macroscopic cracks $\left(\omega^{\varsigma}=1\right)$, the nonlocal domain in the vicinity of the macrocrack should be finally collapsed into the localized discontinuity, i.e. crack line. As noted in Section 3, however, the size of characteristic volume is assumed to be constant and the value of the damage variable in our model is not allowed to reach one to ensure the regularity of the solution. As a result, the localized discontinuity can never be formed and the widening of the damaged zone is unavoidable. In the future work we will explore a transient-gradient damage model [22][23], in which the characteristic length is assumed to be history dependent. As an alternative, we will also consider a possibility of switching to the fracture mechanics approach [32][41], and a unified methodology linking the nonlocal damage theory and fracture mechanics will be also explored [30]. Finally, we notice that our model is proposed for the brittle composite material and interfacial damage is not taken into account.

\section{Acknowledgment}

This work was supported in part by the Allison Engines and the Office of Naval Research through grant number N00014-97-1-0687.

\section{References}

1 Z. P. Bazant (1991), Why continuum damage is nonlocal: micromechanical arguments, J. Engrg. Mech. 117(5), 1070-1087.

2 Z. P. Bazant and G. Pijaudier-Cabot (1988), Nonlocal continuum damage, localization instability and convergence, J. Appl. Mech. 55, 287-293.

3 B. Bhattacharya and B. Ellingwood (1999), A new CDM-based approach to structural deterioration, Int. J. Solids Structures, 36, 1757-1779.

4 N. Bonora and G.M. Newaz (1998), Low cycle fatigue life estimation for ductile metals using a nonlinear continuum damage mechanics model, Int. J. Solids Structures, 35(16), 1881-1894.

5 T. Belytschko and D. Lasry (1989), A study of localization limiters for strain softening in statics and dynamics. Computers and Structures 33, 707-715.

6 T. Belytschko, J. Fish and B. E. Engelman (1988), A finite element with embedded localization zones, Comput. Methods Appl. Mech. Engrg. 70, 59 - 89.

7 A. Benssousan, J. L. Lions and G. Papanicoulau. Asymptotic Analysis for Periodic Structure. NorthHolland, Amsterdam, 1978.

8 E. P. Butler, S.C. Danforth, W.R. Cannon and S.T. Ganczy (1996), Technical Report for the ARPA LC ${ }^{3}$ program, ARPA Agreement No. MDA 972-93-0007.

9 J. L. Chaboche (1988), Continuum damage mechanics I: General concepts \& II: Damage growth, crack initiation and crack growth. J. Appl. Mech. 55, 59-72.

10 J. L. Chaboche and P. M. Lesne (1988), A nonlinear continuous fatigue damage model, Fatigue Fract. 
Engrg. Mater. Struct. 11(1), 1-17.

11 T. L. Chaboche, O. Lesne and T. Pottier (1998), Continuum damage mechanics of composites: towards a unified approach, In: Damage Mechanics in Engineering Materials (Edited by G. Z. Voyiadjis, J. W. Ju and J. L. Chaboche), pp 3-26, Elsevier Science Ltd, Oxford, UK.

12 C. L. Chow and Y. Wei (1991), A model of continuum damage mechanics for fatigue failure, Int. J. Fatigue 50, 301-306.

13 C. L. Chow and Y. Wei (1994), A fatigue damage model for crack propagation, In: Advances in Fatigue Life time Predictive Techniques, ASTM STP 1292 (Edited by M.R. Mitchell and R.W. Landgraf), pp 86-99, American Society of Testing and Materials, Philadelphia, PA.

14 R. H. Dauskardt and R. O. Ritchie (1991), Cyclic fatigue of ceramics, In: Fatigue of Adavanced Materials, (Edited by R. O. Ritchie, R. H. Dauskardt and B. N. Cox), pp 133-151, Materials and Component Engineering Publications Ltd., Birmingham, UK.

15 F. Ellyin, Fatigue Damage, Crack Growth and Life Prediction, Chapman \& Hall, London, UK, 1997.

16 A. Fatemi and L. Yang (1998), Cumulative fatigue damage and life prediction theories: a survey of the start of the art for homogeneous materials, Int. J. Fatigue, 20(1), 9-34.

17 J. Fish, K. Shek, M. Pandheeradi and M. S. Shephard (1997), Computational plasticity for composite structures based on mathematical homogenization: Theory and practice, Comput. Meth. Appl. Mech. Engrg. 148, 53-73.

18 J. Fish and A. Wagiman (1993), Multiscale finite element method for locally nonperiodic heterogeneous medium, Comput. Mech. 12, 164-180.

19 J. Fish and T. Belytschko (1990), A finite element with a unidirectionally enriched strain field for localization analysis, Comput. Methods Appl. Mech. Engrg. 78(2), 181-200.

20 J. Fish, Q. Yu and K. Shek (1999), Computational damage mechanics for composite materials based on mathematical homogenization, Int. J. Numer. Methods Engrg. 45, 1657-1679.

21 J. Fish and Q.Yu (2001), Multiscale Damage Modeling for Composite Materials: Theory and Compuational Framework, Int. J. Numer. Methods Engrg. in print.

22 M. G. D. Geers, Experimental and Computational Modeling of Damage and Fracture. Ph.D Thesis, Technische Universiteit Eindhoven, The Netherlands, 1997.

23 M. G. D. Geers, R. H. J. Peerlings, R. de Borst and W. A. M. Brekelmans (1998), Higher-order damage models for the analysis of fracture in quasi-brittle materials, In: Material Instabilities in Solids, (Edited by R. deBorst and E. van der Giessen), pp 405-423, John Wiley \& Sons, Chichester, UK.

24 J. M. Guedes and N. Kikuchi (1990), Preprocessing and postprocessing for materials based on the homogenization method with adaptive finite element methods, Comput. Methods Appl. Mech. Engrg. 83, 143-198.

25 J. W. Ju (1989) On energy-based coupled elastoplastic damage theories: Constitutive modeling and 
computational aspects. Int. J. Solids Structures 25(7), 803-833.

26 D. Krajcinovic, Damage Mechanics. Elsevier Science Ltd, Oxford, UK, 1996.

27 J. Lemaitre and J. Dufailly (1987), Damage measurements, Enrg. Fract. Mech. 28(5/6), 643-881.

28 S. S. Manson and G. R. Halford (1986), Re-examination of cumulative fatigue damage analysis: An engineering perspective, Enrg. Fract. Mech. 25, 539-571.

29 J. J. Marigo (1985), Modelling of brittle and fatigue damage for elastic material by growth of microvoids, Engrg. Fract. Mech. 21(4), 861-874.

30 J. Mazars and G. Pijaudier-Cabot (1996), From damage to fracture mechanics and conversely: A combined approach, Int. J. Solids Structures 33(20-22), 3327-3342.

31 A. J. McEvily (1988), On crack closure in fatigue crack growth, In: Mechanics of Fatigue Crack Closure, ASTM STP 982, (Edited by J.C. Newman Jr. and W. Elber), pp 35-43, American Society of Testing and Materials, Philadelphia, PA.

32 J. C. Newman Jr. (1997), The merging of fatigue and fracture mechanics concept: a historical respective, In: Fatigue and Fracture Mechanics: 28th Volume, ASTM ASTP 1321, (Edited by J. H. Underwood, B. D. MacDonald and M. R. Ritchell), American Society of Testing and Materials, Philadelphia, PA.

33 Nemat-Nasser, M. Hori, Micromechanics: Overall Properties of Heterogeneous Materials, North-Holland, Amsterdam, The Netherlands, 1993.

34 F. K. G. Odqvist, Mathematical theory of creep and creep rupture, Clarendon Press, Oxford, UK, 1974.

35 E. Papa (1993), A damage model for concrete subjected to fatigue loading, Eur. J. Mech. A/Solids $12(3), 429-440$.

36 M. H. J. W. Pass, P. J. G. Schreurs and W. A. M. Brekelmans (1993), A continuum approach to brittle and fatigue damage: Theory and numerical procedures, Int. J. Solids Structures 30(4), 579-599.

37 R. H. J. Peerling, R. de Borst, W. A. M. Brekelmans and J. H. P. de Vree (1996), Gradient enhanced damage for quasi-brittle materials, Int. J. Numer. Methods Engrg. 39, 3391-3403.

38 E. Sanchez-Palencia, Non-Homogeneous Media and Vibration Theory, Springer-Verlag, Berlin, 1980.

39 J. C. Simo and J. W. Ju (1987), Strain- and stress-based continuum damage models - I. Formulation, Int. J. Solids Structures 23(7), 821-840.

40 J. Stoer and R. Bulirsch, Introduction to Numerical Analysis (Second Edition), Springer-Verlag, New York, 1992.

41 S. Suresh, Fatigue of Materials, Cambridge University Press, Cambridge, UK, 1991

42 R. Talreja, Fatigue of Composite Materials, Technomic Publishing Company, Inc., Lancaster, PA, 1987.

43 R. Talreja (1989), Damage development in composite: Mechanism and modeling, J. Strain Analysis 
$24,215-222$.

44 G. Z. Voyiadjis and T. Park (1996), Elasto-plastic stress and strain concentration tensors for damaged fibrous composites, In: Damage and Interfacial Debonding in Composites (Edited by G. Z. Voyiadjis and D. H. Allen), pp 81-106, Elsevier Science, London, UK.

45 J.C.W. van Vroonhoven and R. deBorst (1999), Combination of fracture and damage mechanics for numerical failure analysis, Int. J. Solids Structures 36, 1169-1191.

\section{Appendix}

In the Appendix we present the details of the derivations of two derivatives, $\partial \boldsymbol{N}^{(m)} / \partial \omega^{(m)}$ in (43) and $\dot{\omega}^{(m)}$ in (49). We start with the first. Taking derivative of (42) gives

$$
\begin{aligned}
\frac{\partial \aleph^{(m)}}{\partial \omega^{(m)}} & =1+\left(\frac{\gamma^{(m)}}{\omega^{(m)}}\right)\left[\frac{\Phi^{(m)}}{\omega^{(m)}}\right]^{\gamma^{(m)}} \frac{\partial \Phi^{(m)}}{\partial \bar{\vartheta}^{(m)}}\left(\bar{\vartheta}^{(m)}-\bar{\vartheta}^{-(m)}\right)- \\
& \left(\frac{1}{\omega^{(m)}}\right)^{\gamma^{(m)}}\left[\gamma^{(m)}\left(\Phi^{(m)}\right)^{\gamma^{(m)}-1}\left(\frac{\partial \Phi^{(m)}}{\partial \bar{\vartheta}^{-(m)}}\right)^{2}+\left(\Phi^{(m)}\right)^{\gamma^{(m)}}\left(\frac{\partial^{2} \Phi^{(m)}}{\partial \vartheta^{-(m) 2}}\right)\right] \frac{\partial \vartheta^{(m)}}{\partial \omega^{(m)}}\left(\bar{\vartheta}^{(m)}-{ }_{t} \vartheta^{-(m)}\right)- \\
& \left(-\left[\frac{\Phi^{(m)}}{\omega^{(m)}}\right]^{\gamma^{(m)}} \frac{\partial \Phi^{(m)}}{\partial \vartheta^{-(m)}} \frac{\partial \vartheta^{(m)}}{\partial \omega^{(m)}}\right)
\end{aligned}
$$

where the terms, $\frac{\partial \Phi^{(m)}}{\partial \bar{\vartheta}^{-(m)}}, \frac{\partial^{2} \Phi^{(m)}}{\partial \vartheta^{-(m) 2}}$ and $\frac{\partial \bar{\vartheta}^{(m)}}{\partial \omega^{(m)}}$ are subsequently computed. From (32), we can get

$$
\frac{\partial \Phi^{(m)}}{\partial \vartheta^{-(m)}}=\frac{\alpha^{(m)} \vartheta_{0}^{-(m)}}{\left[\pi / 2+\operatorname{atan}\left(\beta^{(m)}\right)\right] \cdot\left[\left(\vartheta_{0}^{-(m)}\right)^{2}+\left(\alpha^{(m)} \vartheta^{-(m)}-\beta^{(m)} \vartheta_{0}^{-(m)}\right)^{2}\right]}
$$

and

$$
\frac{\partial^{2} \Phi^{(m)}}{\partial \vartheta^{-(m) 2}}=-\frac{-2 \alpha^{(m)}\left(\alpha^{(m)} \vartheta^{(m)}-\beta^{(m)} \vartheta_{0}^{-(m)}\right)}{\left(\vartheta_{0}^{(m)}\right)^{2}+\left(\alpha^{(m)} \vartheta^{-(m)}-\beta^{(m)} \vartheta_{0}^{-(m)}\right)^{2}} \cdot \frac{\partial \Phi^{(m)}}{\partial \vartheta^{-(m)}}
$$

The derivation of $\frac{\partial \bar{\vartheta}^{(m)}}{\partial \omega^{(m)}}$ is not trivial since the principal components of the nonlocal matrix strains are used to define the matrix damage equivalent strain $\bar{\vartheta}^{(m)}$ in (36). Differentiating (37) with respect to $\omega^{(m)}$ yields

$$
\frac{\partial \bar{\vartheta}^{(m)}}{\partial \omega^{(m)}}=\left(\boldsymbol{b}^{(m)}\right)^{T} \frac{\partial\left(\boldsymbol{F}^{(m)} \hat{\boldsymbol{\varepsilon}}^{(m)}\right)}{\partial \omega^{(m)}}
$$


where the vector $\boldsymbol{b}^{(m)}$ takes following form

$$
\left(\boldsymbol{b}^{(m)}\right)^{T}=\frac{1}{2 \bar{\vartheta}^{(m)}}\left(\boldsymbol{F}^{(m)} \hat{\boldsymbol{\varepsilon}}^{(m)}\right)^{T} \hat{\boldsymbol{L}}^{(m)}
$$

and by using the definition of $\boldsymbol{F}^{(m)}$ in (38), the derivative in (A4) can be expressed as

$$
\frac{\partial\left(\boldsymbol{F}^{(m)} \hat{\boldsymbol{\varepsilon}}^{(m)}\right)}{\partial \omega^{(m)}}=\left[\begin{array}{lll}
\frac{\partial\left(h_{1}^{(m)} \hat{\boldsymbol{\varepsilon}}_{1}^{(m)}\right)}{\partial \omega^{(m)}} & \frac{\partial\left(h_{2}^{(m)} \hat{\boldsymbol{\varepsilon}}_{2}^{(m)}\right)}{\partial \omega^{(m)}} & \frac{\partial\left(h_{3}^{(m)} \hat{\boldsymbol{\varepsilon}}_{3}^{(m)}\right)}{\partial \omega^{(m)}}
\end{array}\right]^{T}
$$

Since the three vector components in (A6) have the same structure, we denote them as $\frac{\partial\left(h_{\xi}^{(m)} \hat{\varepsilon}_{\xi}^{(m)}\right)}{\partial \omega^{(m)}}$ with $\xi=1,2,3$ and then by using (38) we have

$$
\begin{gathered}
\frac{\partial\left(h_{\xi}^{(m)} \hat{\varepsilon}_{\xi}^{(m)}\right)}{\partial \omega^{(m)}}=\left[\frac{\partial h_{\xi}^{(m)}}{\partial \hat{\varepsilon} \xi^{(m)}} \hat{\varepsilon}_{\xi}^{(m)}+h_{\xi}^{(m)}\right] \cdot \frac{\partial \hat{\varepsilon}_{\xi}^{(m)}}{\partial \omega^{(m)}} ; \quad \xi=1,2,3 \\
\frac{\partial \hat{h}_{\xi}^{(m)}}{\partial \hat{\varepsilon}_{\xi}^{(m)}}=\frac{a_{1} / \pi}{1+a_{1}^{2}\left(\hat{\varepsilon}_{\xi}^{(m)}-a_{2}\right)^{2}}
\end{gathered}
$$

To this end we need to compute the derivative of each component of the principal strain $\hat{\varepsilon}^{(m)}$ with respect to the nonlocal damage parameter $\omega^{(m)}$. The principal components of a second order tensor satisfy Hamilton's Theorem, i.e.

$$
\left(\hat{\varepsilon}_{\xi}^{(m)}\right)^{3}-I_{1}\left(\hat{\varepsilon}_{\xi}^{(m)}\right)^{2}+I_{2} \hat{\varepsilon}_{\xi}^{(m)}-I_{3}=0
$$

where $I_{1}, I_{2}, I_{3}$ are the three invariants of $\varepsilon_{i j}^{(m)}$ or $\hat{\varepsilon}^{(m)}$ which can be expressed as

$$
\begin{gathered}
I_{1}=\varepsilon_{i i}^{(m)}=\hat{\varepsilon}_{1}^{(m)}+\hat{\varepsilon}_{2}^{(m)}+\hat{\varepsilon}_{3}^{(m)} \\
I_{2}=\frac{1}{2} \cdot\left(\varepsilon_{i i}^{(m)} \varepsilon_{j j}^{(m)}-\varepsilon_{i j}^{(m)} \varepsilon_{j i}^{(m)}\right)=\hat{\varepsilon}_{1}^{(m) \hat{\varepsilon}} \hat{\varepsilon}^{(m)}+\hat{\varepsilon}_{2}^{(m)} \hat{\varepsilon}_{3}^{(m)}+\hat{\varepsilon}_{3}^{(m)} \hat{\varepsilon}_{1}^{(m)} \\
I_{3}=\frac{1}{6} \cdot\left(2 \varepsilon_{i j}^{(m)} \varepsilon_{j k}^{(m)} \varepsilon_{k i}^{(m)}-3 \varepsilon_{i j}^{(m)} \varepsilon_{j i}^{(m)} \varepsilon_{k k}^{(m)}+\varepsilon_{i i}^{(m)} \varepsilon_{j j}^{(m)} \varepsilon_{k k}^{(m)}\right)=\hat{\varepsilon}_{1}^{(m)} \hat{\varepsilon}_{2}^{(m)} \hat{\varepsilon}_{3}^{(m)}
\end{gathered}
$$

Differentiating (A9) with respect to $\omega^{(m)}$ gives

$$
\frac{\partial \hat{\varepsilon}_{\xi}^{(m)}}{\partial \omega^{(m)}}=\left[3\left(\hat{\varepsilon}_{\xi}^{(m)}\right)^{2}-2 I_{1} \hat{\varepsilon}_{\xi}^{(m)}+I_{2}\right]^{-1} \cdot\left[\frac{\partial I_{1}}{\partial \omega^{(m)}}\left(\hat{\varepsilon}_{\xi}^{(m)}\right)^{2}-\frac{\partial I_{2}}{\partial \omega^{(m)}} \hat{\varepsilon}_{\xi}^{(m)}+\frac{\partial I_{3}}{\partial \omega^{(m)}}\right]
$$


where the derivative of the invariants with respect to $\omega^{(m)}$ can be obtained by using (A10)-(A12)

$$
\begin{gathered}
\frac{\partial I_{1}}{\partial \omega^{(m)}} \equiv E_{i j}^{[1]} \frac{\partial \varepsilon_{i j}^{(m)}}{\partial \omega^{(m)}}=\delta_{i k} \delta_{j k} \frac{\partial \varepsilon_{i j}^{(m)}}{\partial \omega^{(m)}} \\
\frac{\partial I_{2}}{\partial \omega^{(m)}} \equiv E_{i j}^{[2]} \frac{\partial \varepsilon_{i j}^{(m)}}{\partial \omega^{(m)}}=\left(\varepsilon_{m m}^{(m)} \delta_{i k} \delta_{j k}-\varepsilon_{i j}^{(m)}\right) \frac{\partial \varepsilon_{i j}^{(m)}}{\partial \omega^{(m)}} \\
\frac{\partial I_{3}}{\partial \omega^{(m)}} \equiv E_{i j}^{[3]} \frac{\partial \varepsilon_{i j}^{(m)}}{\partial \omega^{(m)}}=\left(\varepsilon_{i k}^{(m)} \varepsilon_{k j}^{(m)}-\varepsilon_{m m}^{(m)} \varepsilon_{i j}^{(m)}-\frac{1}{2} \varepsilon_{m n}^{(m)} \varepsilon_{n m}^{(m)} \delta_{i k} \delta_{j k}+\frac{1}{2} \varepsilon_{m m}^{(m)} \varepsilon_{n n}^{(m)} \delta_{i k} \delta_{j k}\right) \frac{\partial \varepsilon_{i j}^{(m)}}{\partial \omega^{(m)}}
\end{gathered}
$$

Combining (A7), (A13)-(A16), we get

$$
\begin{gathered}
\frac{\partial\left(h_{\xi}^{(m)} \hat{\varepsilon}_{\xi}^{(m)}\right)}{\partial \omega^{(m)}}=Z_{\xi i j}^{(m)} \frac{\partial \varepsilon_{i j}^{(m)}}{\partial \omega^{(m)}} ; \quad \xi=1,2,3 \\
Z_{\xi i j}^{(m)} \equiv\left[\frac{\partial h_{\xi}^{(m)}}{\partial \hat{\varepsilon}_{\xi}^{(m)}} \hat{\varepsilon}_{\xi}^{(m)}+h_{\xi}^{(m)}\right] \cdot\left[3\left(\hat{\varepsilon}_{\xi}^{(m)}\right)^{2}-2 I_{1} \hat{\varepsilon}_{\xi}^{(m)}+I_{2}\right]^{-1} \cdot\left[E_{i j}^{[1]}\left(\hat{\varepsilon}_{\xi}^{(m)}\right)^{2}-E_{i j}^{[2]} \hat{\varepsilon}_{\xi}^{(m)}+E_{i j}^{[3]}\right]
\end{gathered}
$$

Finally the derivative $\partial \bar{\vartheta}^{(m)} / \partial \omega^{(m)}$ in (A4) can be written in a concise form by using (A5) and (A17)

$$
\frac{\partial \bar{\vartheta}^{(m)}}{\partial \omega^{(m)}}=\left(\sum_{\xi=1}^{3} b_{\xi}^{(m)} Z_{\xi i j}^{(m)}\right) \frac{\partial \varepsilon_{i j}^{(m)}}{\partial \omega^{(m)}}
$$

where the derivative on right hand side can be evaluated using (48), i.e.

$$
\frac{\partial \varepsilon_{i j}^{(m)}}{\partial \omega^{(m)}}=G_{i j k l}^{(m)} R_{k l m n}^{(m)} \bar{\varepsilon}_{m n}
$$

To complete the derivations of $\partial \boldsymbol{x}^{(m)} / \partial \omega^{(m)}$ we combine the results of equations (A1)-(A3), (A19) and (A20). The time derivative of the nonlocal matrix damage variable, $\dot{\omega}^{(m)}$, can be obtained by making use of $\partial \aleph^{(m)} / \partial \omega^{(m)}$. From the fatigue damage cumulative law (33), the material derivative of the nonlocal matrix damage parameter (in the case of damage process) can be written as

$$
\dot{\omega}^{(m)}=\left[\frac{\Phi^{(m)}}{\omega^{(m)}}\right]^{\gamma^{(m)}} \frac{\partial \Phi^{(m)} \dot{\dot{\epsilon}}(m)}{\partial \vartheta^{-(m)}}
$$

where $\dot{\bar{\vartheta}}^{(m)}$ is derived in the similar way to $\partial \bar{\vartheta}^{(m)} / \partial \omega^{(m)}$, which yields 


$$
\dot{\bar{\vartheta}}^{(m)}=\left(\sum_{\xi=1}^{3} b_{\xi}^{(m)} Z_{\xi i j}^{(m)}\right) \dot{\varepsilon}_{i j}^{(m)}
$$

where the rate of matrix nonlocal strain can be obtained by taking time derivative of both sides of (18), which gives

$$
\dot{\varepsilon}_{i j}^{(m)}=\left(A_{i j m n}^{(m)}+G_{i j k l}^{(m)} D_{k l m n}\right) \dot{\bar{\varepsilon}}_{m n}+G_{i j k l}^{(m)} R_{k l m n}^{(m)} \bar{\varepsilon}_{m n} \dot{\omega}^{(m)}
$$

Substituting equation (A21) and (A22) into (A23) yields

$$
\dot{\omega}^{(m)} \equiv W_{m n}^{(m)} \dot{\bar{\varepsilon}}_{m n}=\frac{S_{i j}^{(m)}\left(A_{i j m n}^{(m)}+G_{i j k l}^{(m)} D_{k l m n}\right)}{1-S_{i j}^{(m)} G_{i j k l}^{(m)} R_{k l m n}^{(m)} \overline{\bar{\varepsilon}}_{m n}} \dot{\bar{\varepsilon}}_{m n}
$$

where

$$
S_{i j}^{(m)}=\left[\frac{\Phi^{(m)}}{\omega^{(m)}}\right]^{\gamma^{(m)}} \frac{\partial \Phi^{(m)}}{\partial \vartheta^{(m)}}\left(\sum_{\xi=1}^{3} b_{\xi}^{(m)} Z_{\xi i j}^{(m)}\right)
$$

